\title{
BROAD H $\beta$ EMISSION-LINE VARIABILITY IN A SAMPLE OF 102 LOCAL ACTIVE GALAXIES
}

\author{
Jordan N. Runco ${ }^{1}$, Maren Cosens ${ }^{1}$, Vardha N. Bennert ${ }^{1}$, Bryan Scott $^{1}$, S. Komossa ${ }^{2}$, Matthew A. Malkan ${ }^{3}$, \\ Mariana S. Lazarova ${ }^{4}$, Matthew W. Auger ${ }^{5}$, Tommaso Treu ${ }^{3}$, and Daeseong Park ${ }^{6}$ \\ ${ }^{1}$ Physics Department, California Polytechnic State University, San Luis Obispo CA 93407, USA; jrunco@ calpoly.edu, mcosens@ calpoly.edu, vbennert@calpoly.edu \\ ${ }^{2}$ Max-Planck-Institut für Radioastronomie, Auf dem Hügel 69, D-53121, Bonn, Germany \\ ${ }^{3}$ Department of Physics, University of California, Los Angeles, CA 90095, USA; malkan@astro.ucla.edu, tt@ physics.ucsb.edu \\ ${ }^{4}$ Department of Physics and Physical Science, University of Nebraska Kearney, Kearney, NE 68849, USA; lazarovam2@unk.edu \\ 5 Institute of Astronomy, Madingley Road, Cambridge CB3 0HA, UK; mauger@ast.cam.ac.uk \\ ${ }^{6}$ Korea Astronomy and Space Science Institute, Daejeon, 34055, Korea; daeseongpark@kasi.re.kr \\ Received 2015 December 8; accepted 2016 February 28; published 2016 April 5
}

\begin{abstract}
A sample of 102 local $(0.02 \leqslant z \leqslant 0.1)$ Seyfert galaxies with black hole masses $M_{\mathrm{BH}}>10^{7} M_{\odot}$ was selected from the Sloan Digital Sky Survey (SDSS) and observed using the Keck $10 \mathrm{~m}$ telescope to study the scaling relations between $M_{\mathrm{BH}}$ and host galaxy properties. We study profile changes of the broad $\mathrm{H} \beta$ emission line within the three to nine year time frame between the two sets of spectra. The variability of the broad $\mathrm{H} \beta$ emission line is of particular interest, not only because it is used to estimate $M_{\mathrm{BH}}$, but also because its strength and width are used to classify Seyfert galaxies into different types. At least some form of broad-line variability (in either width or flux) is observed in the majority ( $\sim 66 \%$ ) of the objects, resulting in a Seyfert-type change for $\sim 38 \%$ of the objects, likely driven by variable accretion and/or obscuration. The broad $\mathrm{H} \beta$ line virtually disappears in 3/102 ( 3\%) extreme cases. We discuss potential causes for these changing look active galactic nuclei. While similar dramatic transitions have previously been reported in the literature, either on a case-by-case basis or in larger samples focusing on quasars at higher redshifts, our study provides statistical information on the frequency of $\mathrm{H} \beta$ line variability in a sample of low-redshift Seyfert galaxies.
\end{abstract}

Key words: accretion, accretion disks - galaxies: active - galaxies: evolution - galaxies: Seyfert - galaxies: statistics

\section{INTRODUCTION}

Observed relations between the mass of the supermassive black hole $\left(M_{\mathrm{BH}}\right)$ at the center of a galaxy and the properties of its host galaxy-such as host galaxy mass (Magorrian et al. 1998), luminosity (Kormendy \& Richstone 1995), and stellar velocity dispersion (Ferrarese \& Merrit 2000; Gebhardt et al. 2000)-imply a relationship between galaxy evolution and black hole (BH) growth (for a recent review see Kormendy \& Ho 2013; Graham 2016, and references therein). In the local universe, $M_{\mathrm{BH}}$ can be measured by spatially resolving the $\mathrm{BH}$ sphere of influence using stellar or gas kinematics (e.g., van der Marel et al. 1998; Gebhardt et al. 2000). At larger distances the only way to estimate $M_{\mathrm{BH}}$ is by resolving the $\mathrm{BH}$ sphere of influence as it responds to variations in continuum in galaxies with an active galactic nucleus (AGN). In AGNs, the BH is actively growing via an accretion disk of in-falling material. The high-energy photons emitted by the hot accretion disk ionize the surrounding gas clouds, the broad-line region (BLR) in the vicinity of the $\mathrm{BH}$, and the narrow-line region (NLR) further out. Reverberation mapping (Wandel et al. 1999; Kaspi et al. 2005; Bentz et al. 2013) traces variations in the accretion disk continuum luminosity and the time-delayed response of the BLR flux to determine the size of the BLR, using lighttravel time arguments. The velocity of the BLR gas can be determined from the Doppler broadening of the emission lines (such as the broad $\mathrm{H} \beta$ line in the rest-frame optical). By assuming a dimensionless virial coefficient to describe the kinematics and geometry of the BLR, the velocity and size of the BLR combined yield $M_{\mathrm{BH}}$. More recently, there have been attempts to estimate $M_{\mathrm{BH}}$ in individual objects independent of a virial coefficient by modeling reverberation-mapped data directly and constraining the geometry and kinematics of the BLR (see e.g., Pancoast et al. 2014, and references therein).

Seyfert galaxies are low-luminosity AGNs for which the host galaxy can be easily resolved, thus making them attractive targets for the study of the $M_{\mathrm{BH}}$ scaling relations. In fact, for a few Seyfert galaxies, $M_{\mathrm{BH}}$ estimates from both dynamical $\mathrm{AO}$ measurements as well as reverberation mapping are available (Hicks \& Malkan 2008). Seyfert galaxies are categorized into different types based on their emission line profiles, ranging from type-1 to type-2 with subclasses (type-1.5, 1.8, and 1.9) in between. Type-1 Seyferts display both broad and narrow components of emission lines, while type-2 Seyferts show only the narrow components. The intermediate Seyfert types show varying levels of broad component emission. The Balmer series in the optical regime is generally used to classify Seyfert type (Osterbrock \& Koski 1976; Osterbrock 1977, 1981). Table 1 summarizes the different Seyfert-type classifications).

In the framework of the so-called standard unified model for active galaxies, all Seyfert galaxies are thought to be intrinsically the same but viewed from a different angle. The key to this model is a region of cold gas and dust called the dusty torus which surrounds the BLR. If seen edge on, the dusty torus can shield both the accretion disk continuum and the broad emission lines from the observer's view, resulting in a type-2 Seyfert galaxy. If seen face on, however, both accretion disk and BLR are visible, resulting in a type-1 Seyfert galaxy. Intermediate types-1.5, 1.8 , and 1.9 are viewed along the edges of the dusty torus where it is not optically thick enough to fully block the broad lines. In other words, in this simplified model the presence or absence of broad lines is attributed solely to viewing orientation, meaning that the Seyfert type of a galaxy does not change. (Note that the 
Table 1

Seyfert-type Classifications

\begin{tabular}{ll}
\hline \hline $\begin{array}{l}\text { Type } \\
(1)\end{array}$ & $\begin{array}{l}\text { Description } \\
(2)\end{array}$ \\
\hline Type-1 & Both broad and narrow components in all Balmer lines. \\
Type-1.5 & Broad and narrow components can be identified in $\mathrm{H} \alpha$ and $\mathrm{H} \beta$. Broad component of higher-order Balmer lines are weakening. \\
Type-1.8 & Broad $\mathrm{H} \beta$ is weak but detectable. No higher-order Balmer lines have a broad component. \\
Type-1.9 & Shows broad $\mathrm{H} \alpha$ but no higher-order Balmer lines have a broad component. \\
Type-2 & No broad emission lines. \\
\hline
\end{tabular}

Note. Column (1): Seyfert-type. Column (2): Seyfert type classification based on the strength of the $\mathrm{H} \beta$ and $\mathrm{H} \alpha$ lines (Osterbrock 1977, 1981).

orientation of the torus is entirely independent of the host galaxy orientation.)

However, in the literature there have been many reports of apparent Seyfert-type changes (e.g., Tohline \& Osterbrock 1976; Kollatschny \& Fricke 1985; Storchi-Bergmann et al. 1993; Aretxaga et al. 1999; Eracleous \& Halpern 2001; Trippe et al. 2008; Denney et al. 2014; Shappee et al. 2014; Parker et al. 2015). These changes can occur in either direction. NGC 4151 is one of the most notable and cited examples. Originally classified as a type-1.5 (Osterbrock 1977), the broad emission lines disappeared throughout the 1980s (Antonucci \& Cohen 1983; Lyutyi et al. 1984; Penston \& Perez 1984) but have since returned (Shapovalova et al. 2010). Another wellstudied example is Mrk 590, which has been observed over a 40 year timescale. First observed as a type-1.5, Mrk 590 transitioned to a type-1 before the broad lines disappeared, making it a type 1 1.9-2 Seyfert (Denney et al. 2014). Possible causes of these changing look AGNs include changes in extinction (due to our line of sight grazing the dusty torus, for instance; e.g., Goodrich 1989; Leighly et al. 2015), or changes in the AGN accretion rate (e.g., Nicastro 2000; Korista \& Goad 2004; Elitzur et al. 2014) In rare cases, the increase in accretion rate could be due to the tidal disruption and accretion of a star, and a few cases of dramatic broad-line variability possibly linked to this scenario have been reported in recent years (e.g., Komossa et al. 2008; Arcavi et al. 2014; LaMassa et al. 2015; Merloni et al. 2015).

A certain degree of variability in the flux and profile of the BLR emission as a response to changes in the continuum flux (and thus accretion) is not only expected, but in fact forms the basis for reverberation mapping studies. Such studies have shown that variations in continuum flux and that of the broad Balmer lines are correlated in a way so that the derived $M_{\mathrm{BH}}$ does not change (Bentz et al. 2007; Park et al. 2012; Barth et al. 2015). However, extreme variability leading to a type change seems to be rare.

Here we address the question of the frequency of these changing look AGNs by taking advantage of a statistical sample of 102 local Seyfert galaxies with archival spectra from the Sloan Digital Sky Survey (SDSS) and high-quality Keck spectra taken $6.4 \pm 1.8$ years apart. The paper is organized in the following manner. Section 2 summarizes the sample selection, observations, and data reduction. Section 3 describes the analysis of the data. Section 4 discusses the derived quantities and results from the data. Section 5 concludes with a summary. Throughout the paper a Hubble constant of $H_{\mathrm{o}}=70 \mathrm{~km} \mathrm{~s}^{-1}, \Omega_{\lambda}=0.7$, and $\Omega_{\mathrm{M}}=0.3$ is assumed.

\section{SAMPLE SELECTION, OBSERVATIONS, AND DATA REDUCTION}

The primary goal behind sample selection and observations is the creation of a local baseline for the $\mathrm{BH}$ mass scaling relations of active galaxies presented by Bennert et al. (2011, 2015) and Harris et al. (2012), whose papers describe the sample selection, the Keck observations, and the Keck data reduction in detail. Here we provide only a brief summary.

\subsection{Sample Selection}

A sample of 102 local $(0.02 \leqslant z \leqslant 0.1)$ type- 1 Seyfert galaxies was selected from the SDSS data release six (DR6) (Adelman-McCarthy et al. 2008). Objects were selected on the basis of a broad $\mathrm{H} \beta$ emission line with an estimated $M_{\mathrm{BH}}>10^{7} M_{\odot}$ (Bennert et al. 2011; Harris et al. 2012). Note that of these 102 objects, only 79 are used by Bennert et al. (2015) to study the $M_{\mathrm{BH}}-\sigma$ relation because the necessary quantities (i.e., $\lambda L_{5100}, M_{\mathrm{BH}}, \sigma$ ) for that study were only accessible for these 79 objects.

\subsection{SDSS Observations and Data Reduction}

SDSS spectra are obtained from a $2.5 \mathrm{~m}$ ground-based telescope with a $3^{\prime \prime}$ diameter circular optical fiber and an exposure time of $54 \mathrm{~s}$. SDSS spectra cover a wavelength range of 3800-9200 $\AA$ with an instrumental resolution of $170 \mathrm{~km} \mathrm{~s}^{-1}$. SDSS data are already fully reduced and flux calibrated when retrieved from the SDSS archive.

\subsection{Keck Observations and Data Reduction}

The 102 objects selected from SDSS were observed again between 2009 January and 2010 March with the Low Resolution Imaging Spectrometer (LRIS) at the Keck $10 \mathrm{~m}$ telescope using a $1^{\prime \prime} \times 2^{\prime \prime}$ wide rectangular longslit aligned with the major axis of the host galaxy (given by SDSS). While all objects were observed at as low an airmass as possible, given observation constraints the airmass for individual objects can be as high as 1.4. Objects observed in 2009 used a D560 dichroic and objects observed in 2010 used a D680 dichroic. The blue Keck spectra were taken with the 600/400 grism giving a wavelength range of $\sim 3200-5350 \AA$ and an instrumental resolution of $\sim 90 \mathrm{~km} \mathrm{~s}^{-1}$; the red spectra were taken with the $831 / 8200$ grating centered on $8950 \AA$ with a resolution of $\sim 45 \mathrm{~km} \mathrm{~s}^{-1}$. (Note that the red Keck spectra are not used in this paper since they only cover the $\mathrm{Ca}$ triplet absorption lines for an accurate measurement of $\sigma$ ). The exposure times generally range from 600 to $1200 \mathrm{~s}$. Keck 
spectra were taken on average $6.4 \pm 1.8$ years after the SDSS spectra, ranging from 2.6 to 9.1 years (see Table 2 for details on SDSS and Keck observations).

The Keck data are reduced following standard reduction steps such as bias subtraction, flat field correcting, cosmic ray rejection, and wavelength calibration. AOV Hipparcos stars were used to correct for telluric absorption and relative flux calibration. Note that unlike SDSS spectra, Keck spectra are not absolute flux calibrated because observing conditions were typically not photometric. 1D spectra were extracted from the 2D spectra with a width of 1".08 (8 pixels) to encompass the BLR, given the slit width of $1^{\prime \prime}$ and a typical seeing of $1^{\prime \prime}$.

\subsection{Lick Observations and Data Reduction}

For eight objects with significantly weaker or apparently absent broad $\mathrm{H} \beta$ emission in the Keck spectra, follow-up observations were conducted in 2013 January and March with the $3 \mathrm{~m}$ Shane telescope of Lick observatory using the Kast spectrograph and 60 minutes total exposure time per object. (Table 2; Scott 2013). The slit was aligned either along the major axis or perpendicular to it. 1D spectra were extracted using a 4 pixel $\left(\approx 3^{\prime \prime}\right)$ width centered on the peak flux to mimic the $3^{\prime \prime}$ diameter circular fiber of SDSS. The data were reduced following standard procedures. The Lick spectra are presented by Scott (2013). These spectra are used to determine their Seyfert type, since they also cover the $\mathrm{H} \alpha$ region. The Seyfert types based on the Lick spectra are listed in Table 2.

\section{ANALYSIS}

In this paper we focus on four different sets of spectra, for short called "Keck subtracted," "Keck unsubtracted," "SDSS subtracted," and "SDSS unsubtracted," as explained in the next two sections.

\subsection{Unsubtracted Spectra}

To classify Seyfert type and perform a qualitative comparison of the $\mathrm{H} \beta$ region, the reduced spectra are used. Throughout the paper these spectra are referred to as the unsubtracted data set.

For a visual comparison of both data sets (Figures 3-6), the Keck spectra were re-binned to match the lower spectral resolution of the SDSS spectra. Moreover, the spectra were normalized to constant $5007 \AA\left[\mathrm{O}_{\mathrm{III}}\right]$ emission, assuming that the $5007 \AA$ [O III] emission line flux is identical in both data sets, given that emission from the extended NLR does not vary over the observed timescales. This scaling also assumes that both spectra integrate the same [O III] emission over the same area, which might not necessarily be the case given the different apertures used. Aligning the long Keck slit along the major axis of the host galaxy may reduce any difference in [O III] flux covered. However, we discuss aperture effects in more detail below.

\subsection{H及 Fitting and Subtracted Spectra}

A multi-component spectral decomposition is used to fit the region around $\mathrm{H} \beta$. The procedure is summarized here briefly (see Park et al. 2015 for details).

First, the observed continuum is modeled and subtracted by fitting a pseudo-continuum consisting of the featureless AGN power-law continuum, host galaxy starlight templates from the

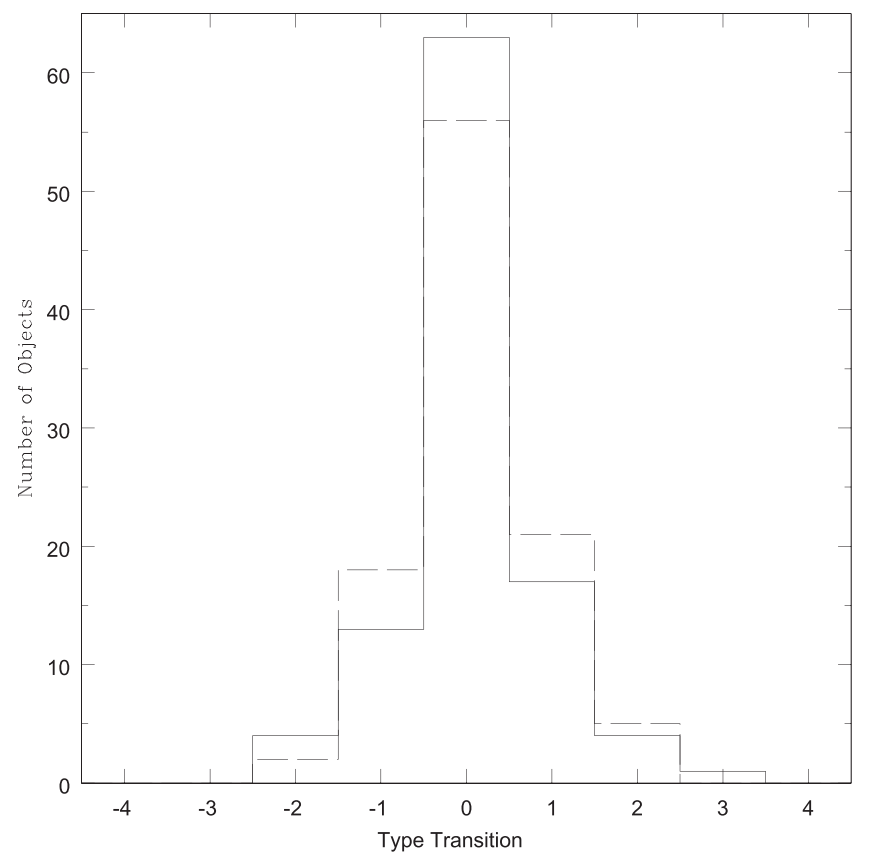

Figure 1. Magnitude of Seyfert-type transitions from visual classification (solid line) and peak flux ratio (dashed line; see the text for details).

Indo-US spectral library (Valdez et al. 2004), and the AGN $\mathrm{Fe}$ II emission template from Boroson \& Green (1992) for the Keck spectra and from Kovacevic et al. (2010) for the SDSS spectra. ${ }^{7}$ Then the continuum-subtracted $\mathrm{H} \beta$ line region is modeled by fitting Gauss-Hermite series (van der Marel \& Franx 1993; Woo et al. 2006; McGill et al. 2008) simultaneously to the $[\mathrm{O} \mathrm{III}]$ narrow emission lines $\lambda \lambda 4959$, $5007 \AA$ and the broad and narrow $\mathrm{H} \beta$ lines, to allow for the fitting of asymmetries. Gauss-Hermite polynomials of order 3-6 are used to fit the broad $\mathrm{H} \beta$ line and 7-12 for each [O III] line. In cases where the He II $\lambda 4686 \AA$ emission line is blended with the broad $\mathrm{H} \beta$, the broad and narrow He II were fitted by simple Gaussian functions.

Depending on the degree of overlap of the broad $\mathrm{H} \beta$ component with the [O $\mathrm{OII}$ lines, we model the $\mathrm{H} \beta$ line region in two slightly different ways. If there is no blending between the broad $\mathrm{H} \beta$ component and the $[\mathrm{O}$ III] $\lambda 55007 \AA$ line, we create a template for the narrow-line components by fitting the [O III] $\lambda 5007 \AA$ line with a Gauss-Hermite series function. The [O III] $\lambda 4959 \AA$ line is then subtracted by blueshifting the template with a flux scale ratio fixed to 1:3 (see, e.g., Dimitrijević et al. 2007, and references therein). Then, the broad and narrow $\mathrm{H} \beta$ components are fitted simultaneously through $\chi^{2}$-minimization, using the blueshifted template from the [O III] $\lambda 5007 \AA$ line as a template for the narrow $\mathrm{H} \beta$, with the flux ratio as a free parameter, and a Gauss-Hermite series for the broad $\mathrm{H} \beta$ component.

If the broad $\mathrm{H} \beta$ component is heavily blended with the [O III] doublet lines, we model the $\mathrm{H} \beta$ broad and narrow lines and the $[\mathrm{O}$ III] $] \lambda 44959,5007 \AA$ lines all together by simultaneously fitting a Gauss-Hermite series function to the broad $\mathrm{H} \beta$

\footnotetext{
7 The two different $\mathrm{Fe}$ II templates are being used due to the different wavelength ranges covered by the Keck and SDSS spectra. The Keck spectra do not extend far enough into the red to fit the Fe II features around 5200 A with the Kovacevic et al. (2010) multi-component template and instead must be fitted with the monolithic template from Boroson \& Green (1992).
} 
component and another Gauss-Hermite series function to the [O III] $] 5007 \AA$ line, where the model for the [O III] $\lambda 5007 \AA$ line is blueshifted and also used for both the [O III] $\lambda 4959 \AA$ line with a 1:3 flux scale ratio and the narrow $\mathrm{H} \beta$ component with a free flux scale ratio. This approach is based on the known fixed flux ratio of [O III] $\lambda \lambda 4959,5007$ of $1: 3$ and the fact that the [O III] lines and the narrow $\mathrm{H} \beta$ lines originate in the NLR and should have comparable widths. It is an approach typically used to fit AGN spectra in, e.g., reverberation mapping studies (Barth et al. 2015; Park et al. 2015).

The results of the spectral fitting are given in Table 3 and the fits are shown in Figures 7-10. The pure emission line spectra -with host galaxy, power-law continuum and Fe II emission subtracted-are referred to in the following as the subtracted data.

Note that for one object in the sample $(1655+2014)$, the signal-to-noise ratio $(\mathrm{S} / \mathrm{N})$ is too low in both the SDSS and the Keck spectra for an accurate measurement of the broad $\mathrm{H} \beta$ line. For three other objects $(0932+0405,0847+1824$, and 0831 +0521 ), the broad $\mathrm{H} \beta$ component could not be accurately identified in the Keck spectra. These four objects are excluded from any discussion involving the $\mathrm{H} \beta$ line fitting.

\subsection{Seyfert-type Classification}

The Seyfert type for all objects was classified independently by eye by two members of the team (J.N.R. and M.C.) following Table 1 , and has been verified independently by the broad $\mathrm{H} \beta$ emission line fitting results. Table 2 lists the Seyferttype classifications for both SDSS and Keck spectra as well as the eight objects observed at Lick. Typically the $\mathrm{H} \alpha$ and $\mathrm{H} \beta$ lines are used for Seyfert-type classification and we followed that procedure for the SDSS and Lick spectra. However, the Keck spectra do not extend to the $\mathrm{H} \alpha$ line. Instead, higherorder Balmer lines $(\mathrm{H} \gamma$ and $\mathrm{H} \delta$ ) were used as a proxy (see Table 1) (Osterbrock 1977). However, these lines are intrinsically much fainter than $\mathrm{H} \alpha$ and $\mathrm{H} \beta$; for example, assuming case $\mathrm{B}$ recombination, $\mathrm{H} \delta(\mathrm{H} \gamma)$ is $\sim 26 \%(47 \%)$ the strength of $\mathrm{H} \beta$ which itself is approximately $35 \%$ of $\mathrm{H} \alpha$ (Osterbrock 1989). Moreover, the $\mathrm{H} \gamma$ line is often blended with the $4383 \AA$ Fe I and the $4363 \AA$ [O III] lines, cautioning the use of this line for classification. Thus, we rely on the $\mathrm{H} \beta$ line for classification. This implies that we cannot differentiate between types-1.9 and 2 for Keck spectra. We conservatively classify an object without a broad $\mathrm{H} \beta$ line in the Keck spectra as a type1.9. Note that given the lower $\mathrm{S} / \mathrm{N}$ of the SDSS spectra, caution should be exercised when classifying the Seyfert subtypes $(1.5,1.8$, and 1.9) since broad lines can be easily lost in the noise. However, given that these are all local Seyfert galaxies, generally, $\mathrm{S} / \mathrm{N}$ are good even for the SDSS spectra. The observed variability of the broad $\mathrm{H} \beta$ line is substantial and the overall trend is for a weaker broad $\mathrm{H} \beta$ line in Keck, partially due to selection effects (see discussion below).

For a more quantitative determination of Seyfert type, we used the $\mathrm{H} \beta$ broad/narrow peak flux ratios and compared them to the visual classification. The reason for choosing peak flux ratios are threefold: (i) using flux ratios eliminates uncertainties on absolute flux calibration; (ii) moreover, compared with integrated flux ratios, peak flux ratios are essentially driving the visual classification scheme; and (iii) the width of the broad $\mathrm{H} \beta$ line can have large uncertainties and depends strongly on the placement of the continuum. Depending on the $\mathrm{S} / \mathrm{N}$ of the data, it can easily be either lost in the noise or noise can be fitted as a
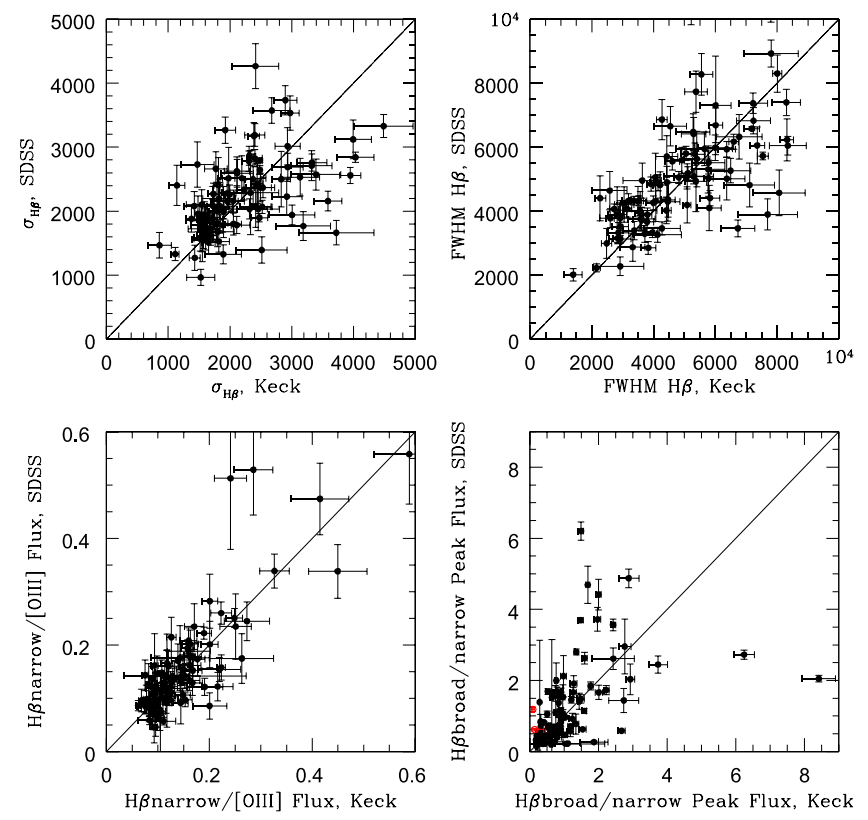

Figure 2. Derived quantities from $\mathrm{H} \beta$ line fitting of SDSS spectra ( $y$-axis) vs. Keck spectra ( $x$-axis; including a unity line). Top left: $\sigma_{\mathrm{H} \beta}$. Top right: FWHM $_{\mathrm{H} \beta}$. Bottom left: $\mathrm{H} \beta_{\text {narrow }} /\left[\mathrm{O}_{\mathrm{III}}\right]$ flux ratio. Bottom right: $\mathrm{H} \beta_{\text {broad }} /$ $\mathrm{H} \beta$ narrow peak flux ratio. The two objects shown in red in this panel were classified as 1.9 in the Keck spectrum, thus they do not have a broad component in $\mathrm{H} \beta$ and this comparison uses an upper limit on the flux if a broad component were included.

broad $\mathrm{H} \beta$ line. Thus, integrated flux ratios are more uncertain than peak flux ratios.

While there is scatter between these two different classification approaches, we determined cutoffs in the $\mathrm{H} \beta$ broad/ narrow peak flux ratios by minimizing the number of outliers. For a peak flux ratio $\geqslant 1.25$, there is an $83 \%$ chance that the object is a type-1; for a $1.25 \geqslant$ peak flux ratio $\geqslant 0.6$, there is a $67 \%$ chance that the object is a type-1.5; and for a peak flux ratio $\leqslant 0.6$, there is a $72 \%$ change that the object is a type-1.8. All type- 1.9 objects have a peak flux ratio of 0 , since there is no broad $\mathrm{H} \beta$ component. In Section 4.1 we discuss the results based on both visual classification as well as peak flux ratios. The peak flux ratios are given in Table 2.

\section{RESULTS AND DISCUSSION}

\subsection{Seyfert-type Transitions}

Based on our visual classifications, at least some degree of type transition is exhibited by $39 / 102$ objects $(39 \pm 10 \%)$. To quantify the "magnitude" of the transition, we assign a value between +4 and -4 in increments of 1 , with a positive value if the broad $\mathrm{H} \beta$ line weakened between SDSS and Keck. Type changes of +4 indicate a full type transition from type- 1 to 2 ; +3 indicates the object transitioned three types (e.g., 1-1.9); +2 indicates a transition of two types (e.g., 1-1.8); and +1 indicates a transition of one type (e.g., 1-1.5); 0 indicates that the object did not experience a Seyfert-type change. A negative value implies that the $\mathrm{H} \beta$ line increased between SDSS and Keck. Figure 1 shows the distribution of the type transitions quantified in this way.

While there are type transitions in either direction, there are more objects that transition toward a narrower/weaker broad $\mathrm{H} \beta$ line in Keck (transition toward type-2) which is likely a reflection of our sample selection, since only Seyfert galaxies 
Table 2

Observations and Seyfert-type Classification

\begin{tabular}{|c|c|c|c|c|c|c|c|c|c|c|c|}
\hline $\begin{array}{l}\text { Object } \\
\text { (1) }\end{array}$ & $\begin{array}{l}\text { R.A. } \\
\text { (J2000) } \\
\text { (2) }\end{array}$ & $\begin{array}{l}\text { decl. } \\
\text { (J2000) } \\
(3)\end{array}$ & $\begin{array}{l}z \\
\text { (4) }\end{array}$ & $\begin{array}{c}\text { Date } \\
\text { SDSS } \\
(5)\end{array}$ & $\begin{array}{c}\text { Class. } \\
\text { SDSS } \\
(6)\end{array}$ & $\begin{array}{c}\text { Date } \\
\text { Keck } \\
(7)\end{array}$ & $\begin{array}{c}\text { Class. } \\
\text { Keck } \\
(8)\end{array}$ & $\begin{array}{c}\text { Exp. Time } \\
\text { Keck (s) } \\
\text { (9) }\end{array}$ & $\begin{array}{c}\text { Diff. } \\
\text { years } \\
(10)\end{array}$ & $\begin{array}{l}\text { Date } \\
\text { Lick } \\
(11)\end{array}$ & $\begin{array}{l}\text { Class. } \\
\text { Lick } \\
(12)\end{array}$ \\
\hline $0013-0951$ & 001335.38 & -095120.9 & 0.062 & $\begin{array}{c}2001 \text { Aug } 17 \\
\text { (52138) }\end{array}$ & 1 & $\begin{array}{c}2009 \text { Sep } 20 \\
(55094)\end{array}$ & 1.5 & 600 & 8.09 & $\cdots$ & $\cdots$ \\
\hline $0026+0009$ & 002621.29 & +00 0914.9 & 0.060 & $\begin{array}{c}2000 \text { Aug } 26 \\
\text { (51782) }\end{array}$ & 1 & $\begin{array}{c}2009 \text { Sep } 20 \\
(55094)\end{array}$ & 1 & 1600 & 9.07 & $\cdots$ & $\ldots$ \\
\hline $0038+0034$ & 003847.96 & +003457.5 & 0.081 & $\begin{array}{c}2000 \text { Sep } 06 \\
(51793)\end{array}$ & 1 & $\begin{array}{c}2009 \text { Sep } 20 \\
(55094)\end{array}$ & 1.5 & 600 & 9.04 & $\cdots$ & $\cdots$ \\
\hline $0109+0059$ & 010939.01 & +00 5950.4 & 0.093 & $\begin{array}{c}2000 \text { Sep } 07 \\
\text { (51794) }\end{array}$ & 1.5 & $\begin{array}{c}2009 \text { Sep } 20 \\
(55094)\end{array}$ & 1.5 & 600 & 9.04 & $\ldots$ & $\cdots$ \\
\hline $0121-0102$ & 012159.81 & -010224.4 & 0.054 & $\begin{array}{c}2000 \text { Sep } 02 \\
(51789)\end{array}$ & 1.5 & $\begin{array}{c}2009 \text { Jan } 21 \\
(54852)\end{array}$ & 1 & 1200 & 8.39 & $\cdots$ & $\cdots$ \\
\hline $0150+0057$ & 015016.43 & +00 5701.9 & 0.085 & $\begin{array}{c}2000 \text { Sep } 06 \\
(51793)\end{array}$ & 1 & $\begin{array}{c}2009 \text { Sep } 20 \\
(55094)\end{array}$ & 1 & 600 & 9.04 & $\ldots$ & $\cdots$ \\
\hline $0206-0017$ & 020615.98 & -00 1729.1 & 0.043 & $\begin{array}{c}2000 \text { Sep } 25 \\
(51812)\end{array}$ & 1 & $\begin{array}{c}2009 \text { Jan } 22 \\
(54853)\end{array}$ & 1 & 1200 & 8.33 & $\cdots$ & $\cdots$ \\
\hline $0212+1406$ & 021257.59 & +140610.0 & 0.062 & $\begin{array}{c}2000 \text { Dec } 05 \\
(51883)\end{array}$ & 1 & $\begin{array}{c}2009 \text { Sep } 20 \\
(55094)\end{array}$ & 1 & 600 & 8.79 & $\cdots$ & $\cdots$ \\
\hline $0301+0110$ & 030124.26 & +011022.5 & 0.072 & $\begin{array}{c}2000 \text { Sep } 30 \\
\text { (51817) }\end{array}$ & 1.5 & $\begin{array}{l}2009 \text { Sep } 20 \\
\text { (55094) }\end{array}$ & 1.5 & 600 & 8.97 & $\cdots$ & $\cdots$ \\
\hline $0301+0115$ & 030144.19 & +011530.8 & 0.075 & $\begin{array}{c}2000 \text { Sep } 30 \\
(51817)\end{array}$ & 1 & $\begin{array}{c}2009 \text { Sep } 20 \\
(55094)\end{array}$ & 1 & 600 & 8.97 & $\cdots$ & $\cdots$ \\
\hline 0310-0049 & 031027.82 & -004950.7 & 0.080 & $\begin{array}{c}2001 \text { Dec } 15 \\
(52258)\end{array}$ & 1 & $\begin{array}{c}2009 \text { Sep } 20 \\
(55094)\end{array}$ & 1 & 600 & 7.76 & $\cdots$ & $\cdots$ \\
\hline 0336-0706 & 033602.09 & -070617.1 & 0.097 & $\begin{array}{c}2000 \text { Dec } 31 \\
(51909)\end{array}$ & 1.8 & $\begin{array}{c}2009 \text { Sep } 20 \\
(55094)\end{array}$ & 1.8 & 2400 & 8.72 & $\cdots$ & $\ldots$ \\
\hline $0353-0623$ & 035301.02 & -062326.3 & 0.076 & $\begin{array}{c}2000 \text { Dec } 30 \\
(51908)\end{array}$ & 1.8 & $\begin{array}{c}2009 \text { Jan } 22 \\
(54853)\end{array}$ & 1 & 1200 & 8.06 & $\cdots$ & $\cdots$ \\
\hline $0731+4522$ & 073126.68 & +452217.4 & 0.092 & $\begin{array}{c}2004 \text { Nov } 05 \\
\text { (53314) }\end{array}$ & 1.5 & $\begin{array}{l}2009 \text { Sep } 20 \\
(55094)\end{array}$ & 1.5 & 600 & 4.87 & $\ldots$ & $\cdots$ \\
\hline $0735+3752$ & 073521.19 & +375201.9 & 0.096 & $\begin{array}{c}2000 \text { Nov } 29 \\
(51877)\end{array}$ & 1.5 & $\begin{array}{l}2009 \text { Sep } 20 \\
\text { (55094) }\end{array}$ & 1.8 & 600 & 8.81 & $\ldots$ & $\ldots$ \\
\hline $0737+4244$ & 073703.28 & +424414.6 & 0.088 & $\begin{array}{c}\text { 2004 Jan } 31 \\
(53035)\end{array}$ & 1.5 & $\begin{array}{c}2009 \text { Sep } 20 \\
\quad(55094)\end{array}$ & 1.5 & 600 & 5.64 & $\cdots$ & $\ldots$ \\
\hline $0802+3104$ & 080243.40 & +310403.3 & 0.041 & $\begin{array}{c}2003 \text { Jan } 02 \\
(52641)\end{array}$ & 1 & $\begin{array}{c}2009 \text { Jan } 21 \\
(54852)\end{array}$ & 1 & 1200 & 5.97 & $\cdots$ & $\cdots$ \\
\hline $0811+1739$ & $\begin{array}{lll}08 & 11 & 10.28\end{array}$ & +173943.9 & 0.065 & $\begin{array}{c}2004 \text { Dec } 18 \\
(53357)\end{array}$ & 1.5 & $\begin{array}{c}2010 \text { Mar } 15 \\
(55270)\end{array}$ & 1 & 2700 & 5.24 & $\cdots$ & $\cdots$ \\
\hline $0813+4608$ & 081319.34 & +460849.5 & 0.054 & $\begin{array}{c}2000 \text { Nov } 29 \\
(51877)\end{array}$ & 1.8 & $\begin{array}{l}2010 \text { Jan } 14 \\
(55210)\end{array}$ & 1 & 1200 & 9.13 & $\cdots$ & $\cdots$ \\
\hline $0831+0521$ & 083107.62 & +052105.9 & 0.035 & $\begin{array}{c}2003 \text { Jan } 07 \\
(52646)\end{array}$ & 1.8 & $\begin{array}{c}2010 \text { Mar } 15 \\
(55270)\end{array}$ & 1.9 & 600 & 7.18 & $\cdots$ & $\cdots$ \\
\hline $0845+3409$ & 084556.67 & +340936.3 & 0.066 & $\begin{array}{c}2003 \mathrm{Feb} 02 \\
(52672)\end{array}$ & 1.5 & $\begin{array}{c}2010 \text { Mar } 14 \\
(55269)\end{array}$ & 1.5 & 3600 & 7.11 & $\cdots$ & $\cdots$ \\
\hline $0846+2522$ & 084654.09 & +252212.3 & 0.051 & $\begin{array}{c}2004 \text { Dec } 19 \\
(53358)\end{array}$ & 1.5 & $\begin{array}{c}2009 \text { Jan } 22 \\
(54853)\end{array}$ & 1.5 & 1200 & 4.09 & $\cdots$ & $\cdots$ \\
\hline $0847+1824$ & 084748.28 & +18 2439.9 & 0.085 & $\begin{array}{c}2005 \text { Dec } 07 \\
(53711)\end{array}$ & 1 & $\begin{array}{c}2009 \text { Jan } 21 \\
(54852)\end{array}$ & 1.9 & 1200 & 3.10 & $\begin{array}{c}2013 \text { Jan } 15 \\
(56307)\end{array}$ & 2 \\
\hline $0854+1741$ & 085439.25 & +174122.5 & 0.065 & $\begin{array}{c}2005 \text { Dec } 25 \\
(53729)\end{array}$ & 1.5 & $\begin{array}{c}2010 \text { Mar } 15 \\
(55270)\end{array}$ & 1 & 600 & 4.22 & $\cdots$ & $\cdots$ \\
\hline $0857+0528$ & 085737.77 & +052821.3 & 0.059 & $\begin{array}{c}2003 \text { Jan } 31 \\
(52670)\end{array}$ & 1 & $\begin{array}{l}2010 \text { Jan } 15 \\
(55211)\end{array}$ & 1 & 600 & 6.96 & $\ldots$ & $\ldots$ \\
\hline $0904+5536$ & 090436.95 & +553602.5 & 0.037 & $\begin{array}{c}2000 \text { Dec } 30 \\
(51908)\end{array}$ & 1.5 & $\begin{array}{c}2010 \text { Mar } 14 \\
(55269)\end{array}$ & 1.5 & 600 & 9.20 & $\cdots$ & $\cdots$ \\
\hline $0909+1330$ & $\begin{array}{lll}09 & 02.35\end{array}$ & +13 3019.4 & 0.051 & $\begin{array}{c}2006 \text { Apr } 01 \\
(53826)\end{array}$ & 1.8 & $\begin{array}{c}2010 \text { Jan } 14 \\
(55210)\end{array}$ & 1 & 600 & 3.79 & $\cdots$ & $\ldots$ \\
\hline $0921+1017$ & 092115.55 & +10 1740.9 & 0.039 & $\begin{array}{c}2004 \text { Feb } 15 \\
(53050)\end{array}$ & 1.8 & $\begin{array}{l}2010 \text { Jan } 14 \\
(55210)\end{array}$ & 1.8 & 700 & 5.91 & $\cdots$ & $\cdots$ \\
\hline $0923+2254$ & 092343.00 & +225432.7 & 0.033 & $\begin{array}{c}2005 \text { Dec } 23 \\
(53727)\end{array}$ & 1 & $\begin{array}{c}2010 \text { Jan } 15 \\
(55211)\end{array}$ & 1 & 600 & 4.06 & $\cdots$ & $\cdots$ \\
\hline $0923+2946$ & 092319.73 & +294609.1 & 0.063 & $\begin{array}{c}2005 \text { Jan } 19 \\
(53389)\end{array}$ & 1.8 & $\begin{array}{c}2010 \text { Jan } 15 \\
(55211)\end{array}$ & 1.8 & 600 & 4.99 & $\cdots$ & $\cdots$ \\
\hline $0927+2301$ & 092718.51 & +230112.3 & 0.026 & $\begin{array}{c}2005 \text { Dec } 26 \\
(53730)\end{array}$ & 1.5 & $\begin{array}{c}2010 \text { Jan } 15 \\
(55211)\end{array}$ & 1.5 & 600 & 4.05 & $\ldots$ & $\ldots$ \\
\hline
\end{tabular}


Table 2

(Continued)

\begin{tabular}{|c|c|c|c|c|c|c|c|c|c|c|c|}
\hline $\begin{array}{l}\text { Object } \\
\text { (1) }\end{array}$ & $\begin{array}{l}\text { R.A. } \\
\text { (J2000) } \\
\text { (2) }\end{array}$ & $\begin{array}{l}\text { decl. } \\
\text { (J2000) } \\
(3)\end{array}$ & $\begin{array}{l}z \\
\text { (4) }\end{array}$ & $\begin{array}{c}\text { Date } \\
\text { SDSS } \\
(5)\end{array}$ & $\begin{array}{c}\text { Class. } \\
\text { SDSS } \\
(6)\end{array}$ & $\begin{array}{c}\text { Date } \\
\text { Keck } \\
(7)\end{array}$ & $\begin{array}{c}\text { Class. } \\
\text { Keck } \\
(8)\end{array}$ & $\begin{array}{c}\text { Exp. Time } \\
\text { Keck (s) } \\
\text { (9) }\end{array}$ & $\begin{array}{c}\text { Diff. } \\
\text { years } \\
(10)\end{array}$ & $\begin{array}{l}\text { Date } \\
\text { Lick } \\
(11)\end{array}$ & $\begin{array}{l}\text { Class. } \\
\text { Lick } \\
(12)\end{array}$ \\
\hline $0932+0233$ & 093240.55 & +023332.6 & 0.057 & $\begin{array}{c}2001 \text { Feb } 25 \\
(51965)\end{array}$ & 1.8 & $\begin{array}{c}2010 \text { Jan } 14 \\
(55210)\end{array}$ & 1.5 & 600 & 8.88 & $\ldots$ & $\ldots$ \\
\hline $0932+0405$ & 093259.60 & +040506.0 & 0.059 & $\begin{array}{c}2001 \text { Dec } 21 \\
\quad(52264)\end{array}$ & 1.8 & $\begin{array}{c}2010 \text { Jan } 14 \\
(55210)\end{array}$ & 1.9 & 600 & 8.07 & $\begin{array}{c}2013 \text { Mar } 11 \\
(56362)\end{array}$ & 1.9 \\
\hline $0936+1014$ & 093641.08 & +101415.7 & 0.060 & $\begin{array}{c}2003 \text { Dec } 20 \\
(52993)\end{array}$ & 1.5 & $\begin{array}{c}2010 \text { Mar } 15 \\
(55270)\end{array}$ & 1 & 3600 & 6.23 & $\ldots$ & $\ldots$ \\
\hline $0938+0743$ & 093812.27 & +074340.0 & 0.022 & $\begin{array}{c}2003 \text { Apr } 04 \\
(52733)\end{array}$ & 1 & $\begin{array}{l}2010 \text { Jan } 14 \\
(55210)\end{array}$ & 1.8 & 600 & 6.78 & $\begin{array}{c}2013 \text { Jan } 15 \\
(56307)\end{array}$ & 1.8 \\
\hline $0948+4030$ & 094838.43 & +403043.5 & 0.047 & $\begin{array}{c}2003 \text { Mar } 11 \\
(52709)\end{array}$ & 1 & $\begin{array}{l}2010 \text { Jan } 15 \\
(55211)\end{array}$ & 1.8 & 900 & 6.85 & $\ldots$ & $\cdots$ \\
\hline $1002+2648$ & 100218.79 & +264805.7 & 0.052 & $\begin{array}{c}2006 \text { Jan } 22 \\
(53757)\end{array}$ & 1.8 & $\begin{array}{l}2010 \text { Jan } 15 \\
(55211)\end{array}$ & 1.9 & 600 & 3.98 & $\ldots$ & $\ldots$ \\
\hline $1029+1408$ & 102925.73 & +140823.2 & 0.061 & $\begin{array}{c}2004 \text { Mar } 11 \\
(53075)\end{array}$ & 1.5 & $\begin{array}{l}2010 \text { Jan } 15 \\
(55211)\end{array}$ & 1.5 & 600 & 5.85 & $\cdots$ & $\cdots$ \\
\hline $1029+2728$ & 102901.63 & +272851.2 & 0.038 & $\begin{array}{c}2006 \mathrm{Feb} 28 \\
(53794)\end{array}$ & 1.8 & $\begin{array}{c}2010 \text { Jan } 15 \\
(55211)\end{array}$ & 1.8 & 600 & 3.88 & $\cdots$ & $\cdots$ \\
\hline $1029+4019$ & 102946.80 & +401913.8 & 0.067 & $\begin{array}{c}2004 \operatorname{Jan} 29 \\
(53033)\end{array}$ & 1.5 & $\begin{array}{c}2010 \text { Jan } 14 \\
(55210)\end{array}$ & 1.5 & 600 & 5.96 & $\ldots$ & $\ldots$ \\
\hline $1038+4658$ & 103833.42 & +465806.6 & 0.063 & $\begin{array}{c}2002 \text { Dec } 12 \\
(52620)\end{array}$ & 1.5 & $\begin{array}{c}2010 \text { Jan } 14 \\
\text { (55210) }\end{array}$ & 1.9 & 600 & 7.09 & $\begin{array}{c}2013 \text { Jan } 17 \\
\text { (56309) }\end{array}$ & 1.9 \\
\hline $1042+0414$ & 104252.94 & +041441.1 & 0.052 & $\begin{array}{c}2002 \text { Mar } 06 \\
(52339)\end{array}$ & 1.5 & $\begin{array}{c}2009 \text { Apr } 16 \\
\text { (54937) }\end{array}$ & 1.5 & 1200 & 7.11 & $\cdots$ & $\cdots$ \\
\hline $1043+1105$ & 104326.47 & +110524.3 & 0.048 & $\begin{array}{c}2004 \text { Apr } 20 \\
(53115)\end{array}$ & 1.8 & $\begin{array}{c}2009 \text { Apr } 16 \\
\text { (54937) }\end{array}$ & 1.8 & 600 & 4.99 & $\ldots$ & $\ldots$ \\
\hline $1049+2451$ & 104925.39 & +245123.7 & 0.055 & $\begin{array}{c}2006 \text { Feb } 26 \\
(53792)\end{array}$ & 1 & $\begin{array}{c}2009 \text { Apr } 16 \\
\text { (54937) }\end{array}$ & 1 & 600 & 3.13 & $\ldots$ & $\ldots$ \\
\hline $1058+5259$ & 105828.76 & +525929.0 & 0.068 & $\begin{array}{c}2003 \text { Jan } 13 \\
(52652)\end{array}$ & 1.5 & $\begin{array}{l}2010 \text { Jan } 14 \\
(55210)\end{array}$ & 1.5 & 600 & 7.00 & $\cdots$ & $\cdots$ \\
\hline $1101+1102$ & 110101.78 & +110248.8 & 0.036 & $\begin{array}{c}2004 \text { Apr } 24 \\
(53119)\end{array}$ & 1.5 & $\begin{array}{c}2009 \text { Apr } 16 \\
\text { (54937) }\end{array}$ & 1.8 & 600 & 4.98 & $\cdots$ & $\cdots$ \\
\hline $1104+4334$ & 110456.03 & +433409.1 & 0.049 & $\begin{array}{c}2004 \mathrm{Feb} 18 \\
(53053)\end{array}$ & 1.8 & $\begin{array}{c}2010 \text { Jan } 14 \\
(55210)\end{array}$ & 1.5 & 600 & 5.91 & $\cdots$ & $\cdots$ \\
\hline $1110+1136$ & 111045.97 & +113641.7 & 0.042 & $\begin{array}{c}2004 \text { Mar } 14 \\
(53078)\end{array}$ & 1.5 & $\begin{array}{c}2010 \text { Mar } 15 \\
(55270)\end{array}$ & 1 & 3600 & 6.00 & $\cdots$ & $\cdots$ \\
\hline $1116+4123$ & 111607.65 & +412353.2 & 0.021 & $\begin{array}{c}2003 \mathrm{Dec} 30 \\
\quad(53003)\end{array}$ & 1.8 & $\begin{array}{c}2009 \text { Apr } 15 \\
\text { (54936) }\end{array}$ & 1.8 & 850 & 5.29 & $\ldots$ & $\ldots$ \\
\hline $1118+2827$ & 111853.02 & +28 2757.6 & 0.060 & $\begin{array}{c}2006 \text { Feb } 27 \\
(53793)\end{array}$ & 1.8 & $\begin{array}{l}2010 \text { Jan } 15 \\
(55211)\end{array}$ & 1.9 & 900 & 3.88 & $\begin{array}{c}2013 \text { Mar } 11 \\
(56362)\end{array}$ & 1.8 \\
\hline $1132+1017$ & 113249.28 & +10 1747.4 & 0.044 & $\begin{array}{c}2003 \text { May } 22 \\
(52781)\end{array}$ & 1.5 & $\begin{array}{c}2010 \text { Jan } 15 \\
(55211)\end{array}$ & 1 & 600 & 6.65 & $\cdots$ & $\cdots$ \\
\hline $1137+4826$ & 113704.17 & +482659.2 & 0.054 & $\begin{array}{c}2003 \text { Jan } 03 \\
(52642)\end{array}$ & 1.5 & $\begin{array}{c}2010 \text { Jan } 14 \\
\text { (55210) }\end{array}$ & 1.5 & 600 & 7.03 & $\cdots$ & $\cdots$ \\
\hline $1139+5911$ & 113908.95 & +591154.6 & 0.061 & $\begin{array}{c}2002 \text { May } 15 \\
(52409)\end{array}$ & 1 & $\begin{array}{c}2010 \text { Jan } 14 \\
(55210)\end{array}$ & 1 & 600 & 7.67 & $\ldots$ & $\cdots$ \\
\hline $1140+2307$ & 114054.09 & +230744.4 & 0.035 & $\begin{array}{c}2006 \text { May } 21 \\
\text { (53876) }\end{array}$ & 1.8 & $\begin{array}{c}2010 \text { Jan } 15 \\
(55211)\end{array}$ & 1.8 & 1200 & 3.66 & $\begin{array}{c}2013 \text { Jan } 13 \\
(56305)\end{array}$ & 2 \\
\hline $1143+5941$ & 114344.30 & +594112.4 & 0.063 & $\begin{array}{c}2002 \text { May } 17 \\
(52411)\end{array}$ & 1.5 & $\begin{array}{c}2010 \text { Mar } 14 \\
(55269)\end{array}$ & 1 & 3000 & 7.82 & $\ldots$ & $\ldots$ \\
\hline $1144+3653$ & 114429.88 & +365308.5 & 0.038 & $\begin{array}{c}2005 \text { Mar } 13 \\
(53442)\end{array}$ & 1 & $\begin{array}{c}2009 \text { Apr } 16 \\
\text { (54937) }\end{array}$ & 1 & 600 & 4.09 & $\ldots$ & $\ldots$ \\
\hline $1145+5547$ & 114545.18 & +554759.6 & 0.053 & $\begin{array}{c}2003 \text { Apr } 30 \\
(52759)\end{array}$ & 1 & $\begin{array}{c}2010 \text { Mar } 14 \\
\text { (55269) }\end{array}$ & 1 & 3600 & 6.87 & $\cdots$ & $\cdots$ \\
\hline $1147+0902$ & 114755.08 & +0902 28.8 & 0.069 & $\begin{array}{c}2003 \text { May } 01 \\
(52760)\end{array}$ & 1.5 & $\begin{array}{c}2010 \text { Jan } 15 \\
(55211)\end{array}$ & 1.5 & 600 & 6.71 & $\cdots$ & $\cdots$ \\
\hline $1205+4959$ & 120556.01 & +495956.4 & 0.063 & $\begin{array}{l}2002 \text { Jun } 17 \\
(52442)\end{array}$ & 1.8 & $\begin{array}{c}2010 \text { Jan } 14 \\
(55210)\end{array}$ & 1.8 & 600 & 7.58 & $\cdots$ & $\cdots$ \\
\hline $1206+4244$ & 120626.29 & +424426.1 & 0.052 & $\begin{array}{c}2004 \text { Apr } 25 \\
(53120)\end{array}$ & 1 & $\begin{array}{c}2010 \text { Mar } 14 \\
(55269)\end{array}$ & 1 & 1100 & 5.88 & $\cdots$ & $\cdots$ \\
\hline $1210+3820$ & 121044.27 & +38 2010.3 & 0.023 & $\begin{array}{c}2005 \text { Apr } 13 \\
(53473)\end{array}$ & 1.5 & $\begin{array}{c}2009 \text { Apr } 16 \\
\text { (54937) }\end{array}$ & 1.5 & 600 & 4.01 & $\cdots$ & $\cdots$ \\
\hline $1216+5049$ & 121607.09 & +504930.0 & 0.031 & $\begin{array}{c}2002 \text { May } 19 \\
\text { (52413) }\end{array}$ & 1.8 & $\begin{array}{c}2010 \text { Mar } 14 \\
\text { (55269) }\end{array}$ & 1.8 & 900 & 6.82 & $\ldots$ & $\ldots$ \\
\hline
\end{tabular}


Table 2

(Continued)

\begin{tabular}{|c|c|c|c|c|c|c|c|c|c|c|c|}
\hline $\begin{array}{l}\text { Object } \\
\text { (1) }\end{array}$ & $\begin{array}{l}\text { R.A. } \\
\text { (J2000) } \\
\text { (2) }\end{array}$ & $\begin{array}{l}\text { decl. } \\
\text { (J2000) } \\
\text { (3) }\end{array}$ & $\begin{array}{l}z \\
\text { (4) }\end{array}$ & $\begin{array}{c}\text { Date } \\
\text { SDSS } \\
(5)\end{array}$ & $\begin{array}{c}\text { Class. } \\
\text { SDSS } \\
(6)\end{array}$ & $\begin{array}{c}\text { Date } \\
\text { Keck } \\
(7)\end{array}$ & $\begin{array}{c}\text { Class. } \\
\text { Keck } \\
(8)\end{array}$ & $\begin{array}{c}\text { Exp. Time } \\
\text { Keck (s) } \\
\text { (9) }\end{array}$ & $\begin{array}{c}\text { Diff. } \\
\text { years } \\
(10)\end{array}$ & $\begin{array}{l}\text { Date } \\
\text { Lick } \\
(11)\end{array}$ & $\begin{array}{c}\text { Class. } \\
\text { Lick } \\
(12)\end{array}$ \\
\hline $1223+0240$ & 122324.14 & +024044.4 & 0.024 & $\begin{array}{c}2002 \text { Jan } 09 \\
(52283)\end{array}$ & 1 & $\begin{array}{c}2010 \text { Mar } 15 \\
(55270)\end{array}$ & 1 & 600 & 8.18 & $\ldots$ & $\ldots$ \\
\hline $1228+0951$ & 122811.41 & +095126.7 & 0.064 & $\begin{array}{c}2003 \text { Apr } 02 \\
(52731)\end{array}$ & 1.8 & $\begin{array}{c}2010 \text { Mar } 15 \\
(55270)\end{array}$ & 1.8 & 600 & 6.95 & $\ldots$ & $\ldots$ \\
\hline $1231+4504$ & 123152.04 & +450442.9 & 0.062 & $\begin{array}{c}2004 \mathrm{Feb} 27 \\
(53062)\end{array}$ & 1.5 & $\begin{array}{l}2010 \text { Jan } 15 \\
(55211)\end{array}$ & 1.5 & 1200 & 5.88 & $\ldots$ & $\ldots$ \\
\hline $1241+3722$ & 124129.42 & +372201.9 & 0.063 & $\begin{array}{c}2006 \text { Apr } 02 \\
(53827)\end{array}$ & 1.5 & $\begin{array}{c}2010 \text { Jan } 15 \\
(55211)\end{array}$ & 1.5 & 800 & 3.79 & $\cdots$ & $\ldots$ \\
\hline $1246+5134$ & 124638.74 & +513455.9 & 0.067 & $\begin{array}{c}2002 \text { Apr } 15 \\
(52379)\end{array}$ & 1.8 & $\begin{array}{c}2010 \text { Jan } 15 \\
(55211)\end{array}$ & 1.5 & 600 & 7.75 & $\ldots$ & $\cdots$ \\
\hline $1250-0249$ & 125042.44 & -024931.5 & 0.047 & $\begin{array}{c}2001 \text { Mar } 29 \\
\text { (51997) }\end{array}$ & 1.5 & $\begin{array}{c}2009 \text { Apr } 16 \\
(54937)\end{array}$ & 1.8 & 1200 & 8.05 & $\ldots$ & $\ldots$ \\
\hline $1306+4552$ & 130619.83 & +455224.2 & 0.051 & $\begin{array}{c}2004 \text { Apr } 22 \\
(53117)\end{array}$ & 1 & $\begin{array}{c}2010 \text { Mar } 14 \\
(55269)\end{array}$ & 1.5 & 3600 & 5.89 & $\ldots$ & $\ldots$ \\
\hline $1307+0952$ & 130721.93 & +095209.3 & 0.049 & $\begin{array}{c}2006 \text { May } 29 \\
\text { (53884) }\end{array}$ & 1.8 & $\begin{array}{c}2010 \text { Mar } 15 \\
(55270)\end{array}$ & 1.5 & 2400 & 3.79 & $\ldots$ & $\ldots$ \\
\hline $1312+2628$ & 131259.59 & +262824.0 & 0.060 & $\begin{array}{c}2006 \mathrm{Feb} 28 \\
(53794)\end{array}$ & 1 & $\begin{array}{c}2010 \text { Mar } 14 \\
(55269)\end{array}$ & 1 & 2700 & 4.04 & $\ldots$ & $\ldots$ \\
\hline $1313+3653$ & 131348.96 & +365357.9 & 0.067 & $\begin{array}{c}2006 \text { Mar } 21 \\
(53815)\end{array}$ & 1.8 & $\begin{array}{c}2010 \text { Mar } 14 \\
(55269)\end{array}$ & 1.8 & 600 & 3.98 & $\ldots$ & $\ldots$ \\
\hline $1323+2701$ & 132310.39 & +270140.4 & 0.056 & $\begin{array}{c}2006 \text { Mar } 01 \\
(53795)\end{array}$ & 1.8 & $\begin{array}{c}2009 \text { Apr } 16 \\
(54937)\end{array}$ & 1.8 & 700 & 3.13 & $\ldots$ & $\ldots$ \\
\hline $1353+3951$ & 135345.93 & +395101.6 & 0.063 & $\begin{array}{c}2004 \text { Feb } 26 \\
(53061)\end{array}$ & 1.8 & $\begin{array}{c}2010 \text { Mar } 14 \\
(55269)\end{array}$ & 1.9 & 600 & 6.05 & $\begin{array}{c}2013 \text { Mar } 12 \\
(56363)\end{array}$ & 1.8 \\
\hline $1355+3834$ & 135553.52 & +383428.5 & 0.050 & $\begin{array}{c}2005 \text { Mar } 31 \\
(53460)\end{array}$ & 1.8 & $\begin{array}{c}2009 \text { Apr } 16 \\
\text { (54937) }\end{array}$ & 1.8 & 300 & 4.04 & $\ldots$ & $\ldots$ \\
\hline $1405-0259$ & 140514.86 & -025901.2 & 0.054 & $\begin{array}{c}2002 \text { Jun } 18 \\
(52443)\end{array}$ & 1 & $\begin{array}{c}2009 \text { Apr } 16 \\
(54937)\end{array}$ & 1 & 1600 & 6.83 & $\ldots$ & $\cdots$ \\
\hline $1416+0317$ & 141630.82 & +013707.9 & 0.054 & $\begin{array}{c}2001 \text { Mar } 26 \\
(51994)\end{array}$ & 1.5 & $\begin{array}{c}2010 \text { Mar } 15 \\
(55270)\end{array}$ & 1.8 & 2700 & 8.97 & $\ldots$ & $\cdots$ \\
\hline $1419+0754$ & 141908.30 & +075449.6 & 0.056 & $\begin{array}{c}2005 \text { Jun } 12 \\
(53533)\end{array}$ & 1.8 & $\begin{array}{c}2009 \text { Apr } 16 \\
(54937)\end{array}$ & 1.8 & 900 & 3.84 & $\ldots$ & $\cdots$ \\
\hline $1423+2720$ & 142338.43 & +272009.7 & 0.064 & $\begin{array}{c}2006 \text { May } 26 \\
\text { (53881) }\end{array}$ & 1.5 & $\begin{array}{c}2010 \text { Mar } 14 \\
(55269)\end{array}$ & 1.9 & 1200 & 3.80 & $\begin{array}{c}2013 \text { Mar } 12 \\
(56363)\end{array}$ & 1.8 \\
\hline $1434+4839$ & 143452.45 & +483942.8 & 0.037 & $\begin{array}{c}2003 \text { Apr } 04 \\
(52733)\end{array}$ & 1 & $\begin{array}{c}2009 \text { Apr } 16 \\
(54937)\end{array}$ & 1.5 & 600 & 6.03 & $\ldots$ & $\ldots$ \\
\hline $1505+0342$ & 150556.55 & +034226.3 & 0.036 & $\begin{array}{c}2001 \text { May } 26 \\
(52055)\end{array}$ & 1.5 & $\begin{array}{c}2010 \text { Mar } 15 \\
(55270)\end{array}$ & 1.5 & 1200 & 8.80 & $\ldots$ & $\cdots$ \\
\hline $1535+5754$ & 153552.40 & +575409.3 & 0.030 & $\begin{array}{c}2002 \text { Mar } 14 \\
(52347)\end{array}$ & 1 & $\begin{array}{c}2009 \text { Apr } 15 \\
(54936)\end{array}$ & 1 & 1200 & 7.09 & $\ldots$ & $\ldots$ \\
\hline $1543+3631$ & 154351.49 & +363136.7 & 0.067 & $\begin{array}{l}2003 \text { Aug } 24 \\
\text { (52875) }\end{array}$ & 1 & $\begin{array}{c}2010 \text { Mar } 15 \\
(55270)\end{array}$ & 1.5 & 1200 & 6.56 & $\cdots$ & $\cdots$ \\
\hline $1545+1709$ & 154507.53 & +170951.1 & 0.048 & $\begin{array}{c}2006 \text { Jun } 03 \\
(53889)\end{array}$ & 1.8 & $\begin{array}{c}2009 \text { Apr } 15 \\
\text { (54936) }\end{array}$ & 1 & 1200 & 2.57 & $\ldots$ & $\ldots$ \\
\hline $1554+3238$ & 155417.42 & +323837.6 & 0.048 & $\begin{array}{c}2003 \text { Jul } 05 \\
(52825)\end{array}$ & 1.5 & $\begin{array}{c}2009 \text { Apr } 15 \\
(54936)\end{array}$ & 1.5 & 1200 & 5.78 & $\ldots$ & $\ldots$ \\
\hline $1557+0830$ & 155733.13 & +083042.9 & 0.047 & $\begin{array}{l}2004 \text { Aug } 11 \\
(53228)\end{array}$ & 1.5 & $\begin{array}{c}2009 \text { Apr } 15 \\
(54936)\end{array}$ & 1.5 & 1200 & 4.68 & $\ldots$ & $\ldots$ \\
\hline $1605+3305$ & 160502.46 & +330544.8 & 0.053 & $\begin{array}{c}2004 \text { May } 17 \\
\text { (53142) }\end{array}$ & 1 & $\begin{array}{c}2009 \text { Apr } 15 \\
(54936)\end{array}$ & 1 & 1200 & 4.91 & $\ldots$ & $\ldots$ \\
\hline $1606+3324$ & 160655.94 & +332400.3 & 0.059 & $\begin{array}{c}2004 \text { May } 19 \\
\text { (53144) }\end{array}$ & 1.5 & $\begin{array}{c}2009 \text { Apr } 15 \\
(54936)\end{array}$ & 1.5 & 1200 & 4.91 & $\ldots$ & $\ldots$ \\
\hline $1611+5211$ & 161156.30 & +521116.8 & 0.041 & $\begin{array}{c}2001 \text { May } 22 \\
(52051)\end{array}$ & 1.5 & $\begin{array}{c}2009 \text { Apr } 15 \\
(54936)\end{array}$ & 1.8 & 1200 & 7.95 & $\ldots$ & $\ldots$ \\
\hline $1636+4202$ & 163631.28 & +420242.5 & 0.061 & $\begin{array}{c}2001 \text { Jun } 23 \\
(52083)\end{array}$ & 1 & $\begin{array}{c}2010 \text { Mar } 14 \\
(55269)\end{array}$ & 1 & 1200 & 8.72 & $\ldots$ & $\ldots$ \\
\hline $1647+4442$ & 164721.47 & +44 4209.7 & 0.025 & $\begin{array}{c}2001 \text { May } 22 \\
(52051)\end{array}$ & 1.8 & $\begin{array}{c}2010 \text { Mar } 14 \\
(55269)\end{array}$ & 1.5 & 4200 & 8.81 & $\ldots$ & $\ldots$ \\
\hline $1655+2014$ & 165514.21 & +201442.0 & 0.084 & $\begin{array}{c}2004 \text { Jun } 13 \\
(53169)\end{array}$ & 1.8 & $\begin{array}{c}2009 \text { Sep } 20 \\
(55094)\end{array}$ & 1.8 & 600 & 5.27 & $\ldots$ & $\cdots$ \\
\hline $1708+2153$ & 170859.15 & +215308.1 & 0.072 & $\begin{array}{c}2004 \text { Jun } 21 \\
\text { (53177) }\end{array}$ & 1.5 & $\begin{array}{l}2009 \text { Sep } 20 \\
\text { (55094) }\end{array}$ & 1 & 600 & 5.25 & $\ldots$ & $\ldots$ \\
\hline
\end{tabular}


Table 2

(Continued)

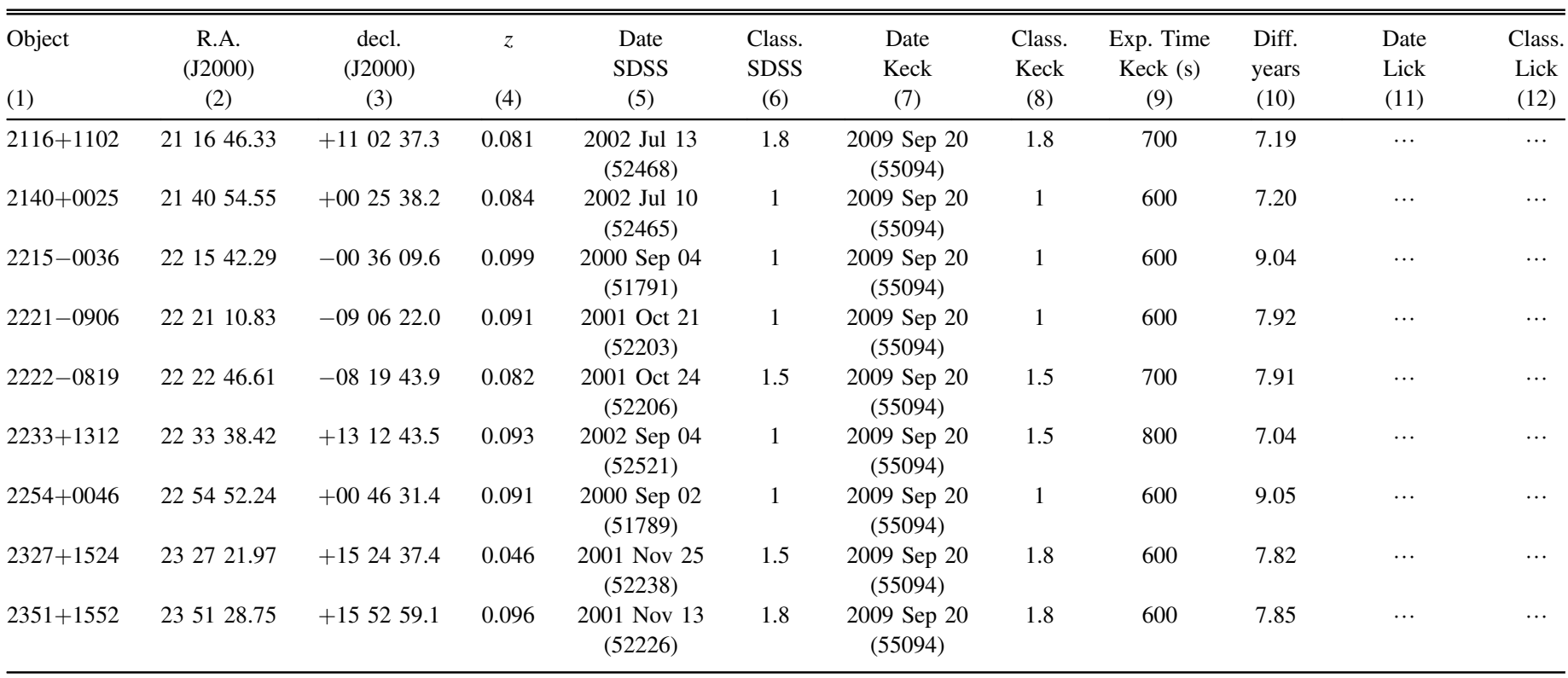

Note. Column (1): target ID based on R.A. and decl. used throughout the text. Column (2): R.A. Column (3): decl. Column (4): redshift from SDSS-DR7. Column (5): date on which SDSS spectrum was taken (with Modified Julian Date (MJD) in brackets). Column (6): Seyfert-type classification based on SDSS spectrum. Column (7): date on which Keck spectrum was taken (with MJD in brackets). Column (8): Seyfert-type classification based on Keck spectrum. Note that because Keck spectra do not extend to $\mathrm{H} \alpha$, we cannot differentiate between type-1.9 and type-2, and conservatively classify these objects in question as type-1.9. Column (9): exposure time of Keck observations in seconds. Note that the exposure time for all SDSS spectra is 54 s. Column (10): time between SDSS and Keck observations in years. Column (11): date on which Lick spectrum was taken (with MJD in brackets). Column (12): Seyfert-type classification based on Lick spectrum.

with a broad $\mathrm{H} \beta$ component were selected from SDSS (Bennert et al. 2011; Harris et al. 2012). Of the 39 objects with a type transition, the majority (31) experiences only a minor type change ( \pm 1 mag transition), while eight underwent greater type changes of 2 . One object $(0847+1824)$ demonstrated a type transition of magnitude 3 . We consider three objects (3\% of the sample) extreme objects since they all show cases of a disappearing broad $\mathrm{H} \beta$ line between SDSS and Keck. We discuss them in detail in Section 4.3. There is no correlation between the magnitude of the type transition and the time between observations, indicating that a Seyfert-type change happens on shorter timescales than those covered by our observations (6.4 \pm 1.8 years).

If we instead use our peak flux ratio cutoffs as discussed in Section 4.1 to classify the Seyfert type, the results change only slightly: 46/102 objects undergo a type transition, with the majority (39) experiencing only a minor type change $( \pm 1$ magnitude transition), while seven underwent greater type changes of 2. However, we consider the visual classification more reliable and it is also commonly used in the literature. Thus, in the following we refer to the visual classification.

As outlined in the introduction, AGN type changes have previously been observed in the literature and are often referred to as "changing look" AGNs (see, e.g., Denney et al. 2014; LaMassa et al. 2015; Runnoe et al. 2016, and references therein); indeed, the frequency and strength of our observed $\mathrm{H} \beta$ emission line variability is in line with one of the first studies on this topic (Rosenblatt et al. 1994).

Two common explanations of type transitions are variable accretion and variable obscuration. Variable accretion, caused by fluctuating amounts of gas available to feed the $\mathrm{BH}$
(Bochkarev 2006), results in a change of the continuum flux and subsequently in a change of the broad $\mathrm{H} \beta$ emission line flux since the BLR clouds are photoionized by the UV continuum. There are many papers citing variable accretion as the driving force behind Seyfert-type transitions (Eracleous \& Halpern 2001; Trippe et al. 2008; Denney et al. 2014; Shappee et al. 2014). Denney et al. (2014) is the most notable example reporting Mrk 590 transitioning from type-1.5 to type-1, then transitioning again to type $\sim 1.9-2$. Shappee et al. (2014) (for NGC 2617), Trippe et al. (2008) (for NGC 2992), and Antonucci \& Cohen (1983) (for NGC 4151) report an observed change in X-ray flux that is followed by a similar change in UV/optical flux. Variable extinction can occur when dusty clouds pass our line of sight. For Seyfert galaxies, the source of this obscuration is likely the dusty torus, thought to surround the BLR in the framework of the standard unified model. The individual cold gas clouds of the dusty torus are not all identical and uniform, so different gas clouds could shield different amounts of continuum and $\mathrm{H} \beta$ flux.

The disappearance of broad lines as seen in 3\% of our sample (discussed in detail in Section 4.3) has also been documented before. In particular, Ho \& Kim (2009) report that ten of the 94 objects in their local sample $(\sim 11 \%)$, selected to investigate relationships between $M_{\mathrm{BH}}$ and host galaxy properties using Magellan spectra (3600-6000 A wavelength range), had only narrow lines when the same objects were previously classified as type-1 Seyferts. (Note that Ho \& Kim 2009 do not discuss this matter further since it was not the main purpose of their paper.)

In a sample of 117 changing look quasar candidates selected from SDSS DR12, Ruan et al. (2015) discover two new low- 


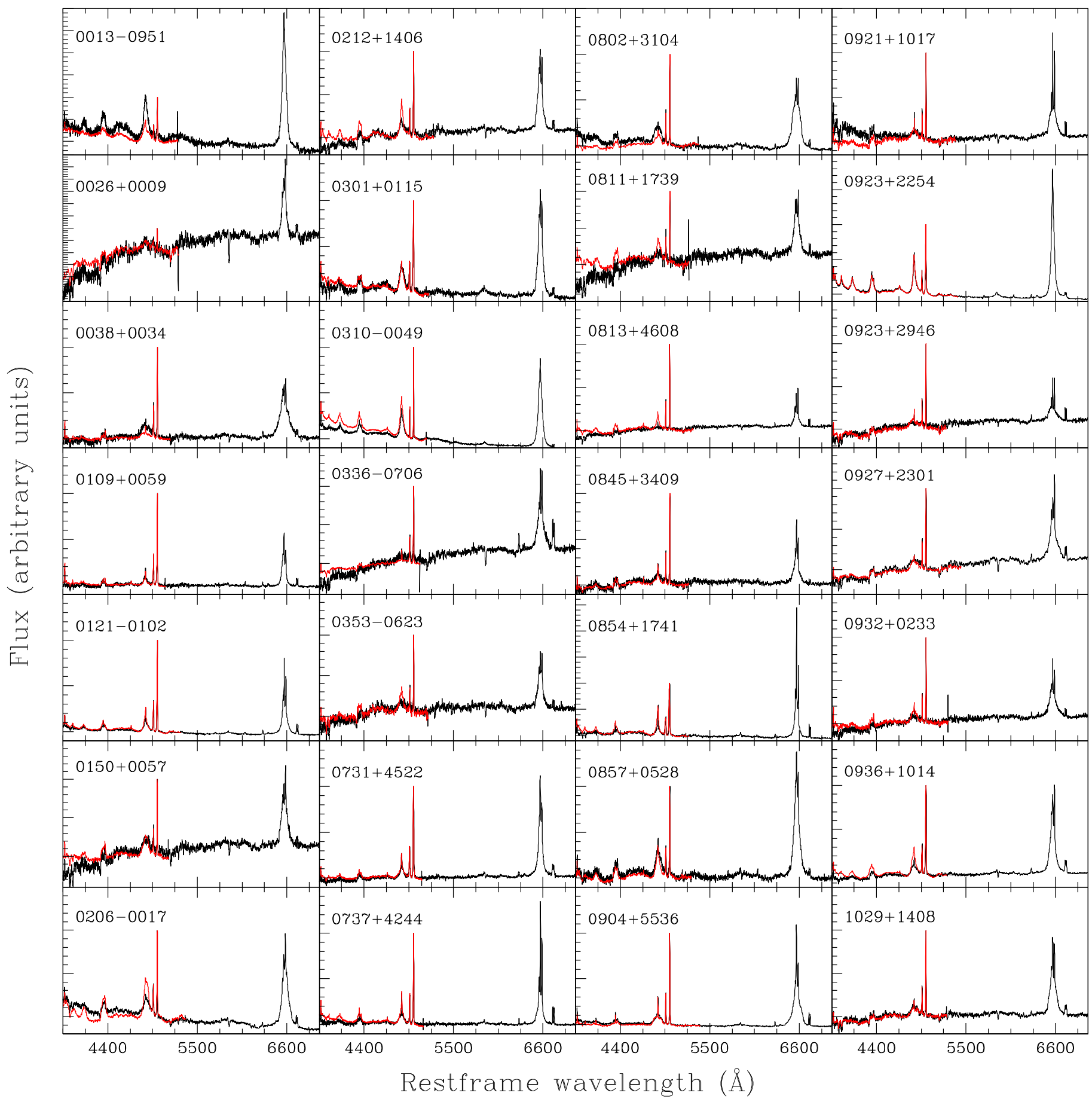

Figure 3. Unsubtracted spectra comparing SDSS (black) and Keck (red) (3850-7000 Å). Note that the Keck spectra only cover the blue part. For comparison, the Keck spectra were re-binned to match the lower spectral resolution of the SDSS spectra. Moreover, for both spectra, the base of the $5007 \AA$ [O mII line was set to 0 and the peak to 1 (see text for discussion). Objects shown in this figure are included in Bennert et al. (2015).

redshift quasars (in addition to the one previously found by LaMassa et al. 2015) where both the broad $\mathrm{H} \beta$ and continuum luminosity dim over the five to seven years in rest-frame time, changing the objects from quasar-like to galaxy-like states. Ruan et al. (2015) argue that the observed change is driven by rapidly decreasing accretion rates.

From a sample of 1011 low-redshift quasars $(z<0.63)$ selected based on repeated photometry from SDSS and PanSTARRS1 as well as repeated spectra from SDSS and SDSSIII Boss, MacLeod et al. (2016) visually identify 10 changing look quasars, with $4 / 10$ of these objects showing emission lines broadening with at least a one magnitude increase in g-band flux and $5 / 10$ of these objects having disappearing broad emission lines and a decreasing lightcurve (one of these five objects was discovered by LaMassa et al. 2015). One of these five objects (SDSS J1021+4645) experienced a transition from type-1 to type-1.9. MacLeod et al. (2016) report significant changes on timescales of 2000-3000 days with broad emission line changes corresponding to continuum changes. Variable accretion and obscuration were both 


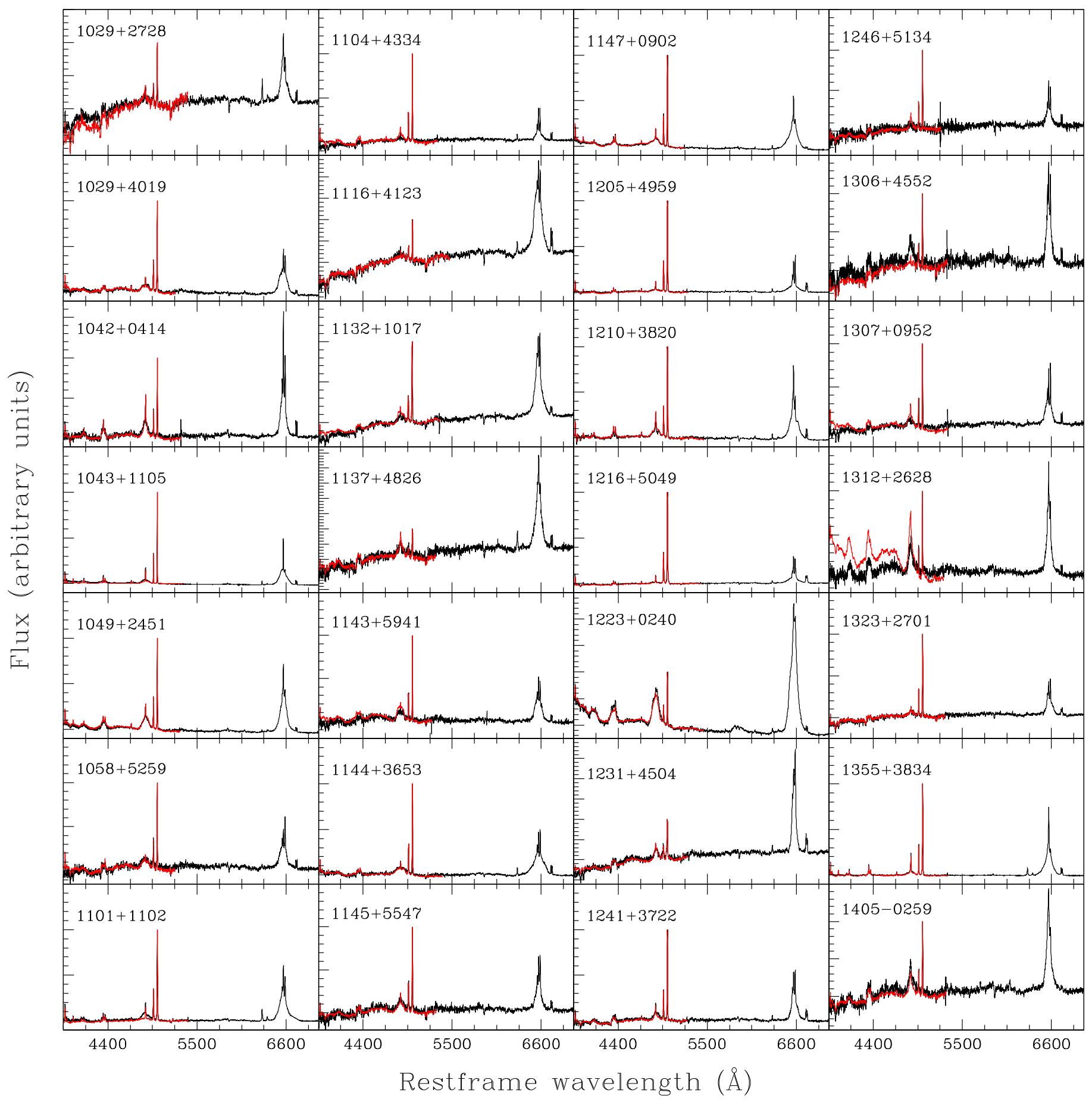

Figure 4. Same as Figure 3.

discussed as possible options to explain the observed broadline changes, and neither possibility was ruled out (MacLeod et al. 2016). A tidal disruption flare event might explain the observed changes behind J0159+0033 (MacLeod et al. 2016; Merloni et al. 2015).

For the purpose of a reverberation mapping campaign, Barth et al. (2015) re-observed AGNs classified as Seyfert 1 galaxies based on SDSS spectra five to eight years later with the $3 \mathrm{~m}$ Shane telescope of Lick observatory and noted that for one object (NGC 6423), all emission lines had disappeared and that three other objects (Mrk 474, Mrk 728, and Mrk 1494) changed from Seyfert 1 to Seyfert 1.9. We will discuss possible explanations for such extreme changes in Section 4.3.

\subsection{Quantifying the Observed Broad H $\beta$-Line Changes}

We use several measurements from our spectral decomposition of the $\mathrm{H} \beta$ region discussed in Section 3.2 to further quantify the observed broad $\mathrm{H} \beta$ line changes and explore possible origins, particularly in these four quantities: (i) the second moment of the broad $\mathrm{H} \beta$ component $\left(\sigma_{\mathbf{H} \beta}\right)$ from the model used to calculate $M_{\mathrm{BH}}$ (Bennert et al. 2011, 2015); (ii) the full width at half maximum (FWHM) of the broad $\mathrm{H} \beta$ line also 


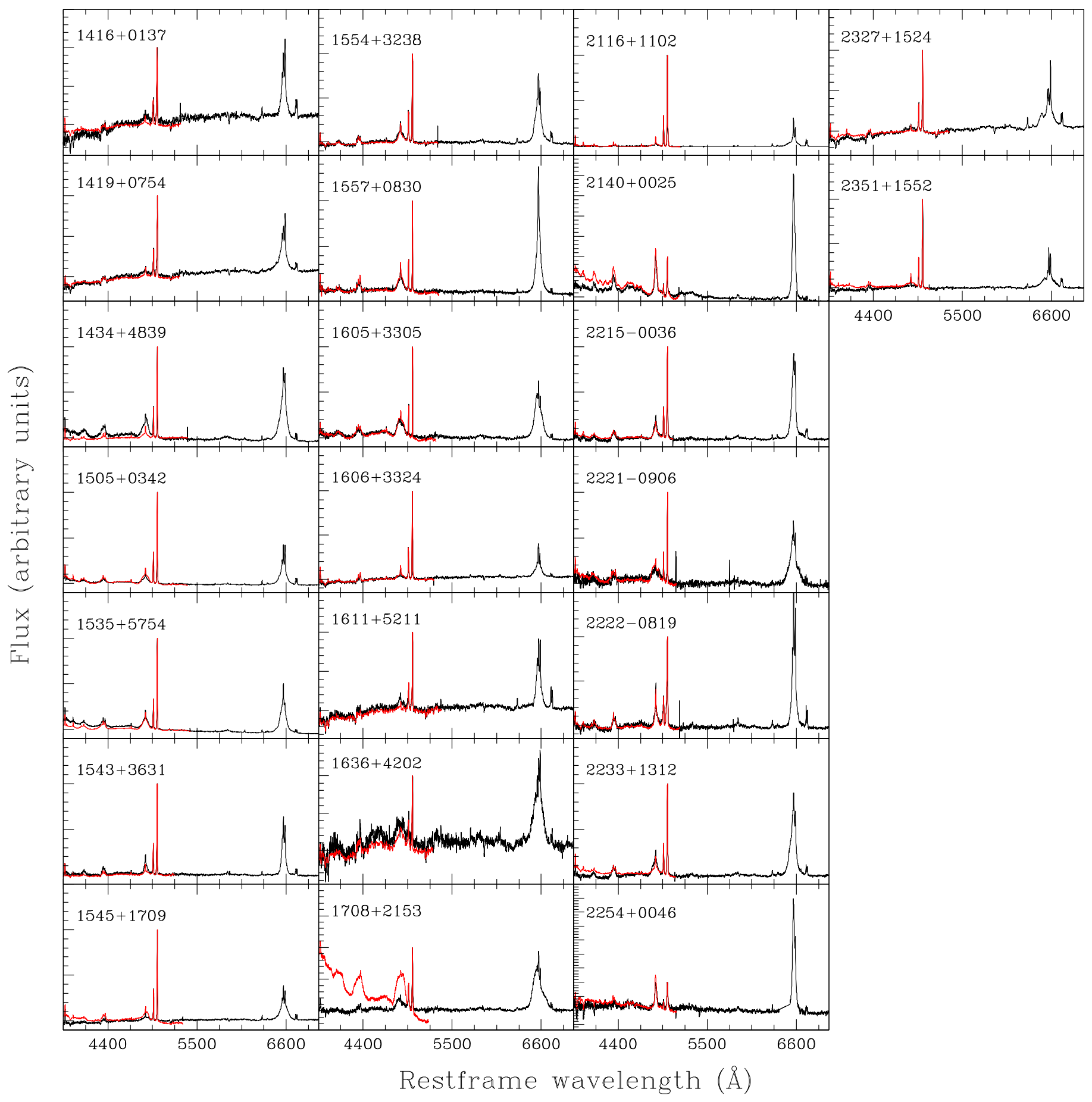

Figure 5. Same as Figure 3.

sometimes used to calculate $M_{\mathrm{BH}}$ (e.g., Shen et al. 2008; Shen et al. 2011) ${ }^{8}$; (iii) the flux ratio of the narrow $\mathrm{H} \beta$ component and [O III] lines, $\mathrm{H} \beta_{\text {narrow }} /[\mathrm{O}$ III]; and (iv) the peak flux ratio of the broad and narrow components of the $\mathrm{H} \beta$ line, $\mathrm{H} \beta_{\text {broad }} / \mathrm{H} \beta_{\text {narrow. }}$. In Figure 2 we compare these four quantities as derived from the SDSS spectra with those derived from the Keck spectra.

On average, the broad $\mathrm{H} \beta$ line is wider in the SDSS spectra than in the Keck spectra, both in $\sigma_{\mathrm{H} \beta}(1.07 \pm 0.29)$ and $\mathrm{FWHM}_{\mathrm{H} \beta}(1.08 \pm 0.27)$ (Table 5). Moreover, the broad $\mathrm{H} \beta$ line has more peak flux in the SDSS spectra than in the Keck

\footnotetext{
$\overline{8 \text { For a Gaussian profile, } \mathrm{FWHM}_{\mathrm{H} \beta}=2.35} \sigma_{\mathrm{H} \beta}$.
}

spectra $(1.26 \pm 0.12)$. The broader and stronger $\mathrm{H} \beta$ line preferentially in the SDSS spectra is likely attributed to the bias in the sample selection since only objects with a broad $\mathrm{H} \beta$ line were chosen from SDSS.

What is noticeable in the top left panel of Figure 2 is the large scatter: for individual objects, $\sigma_{\mathrm{H} \beta}$ can be almost up to a factor of two different between the two sets of spectra. FHWM follows a similar trend. Reverberation mapping studies have shown that the variability of the line width in AGNs correlates inversely with the variability of the power-law continuum in a way that it cancels out in the virial product, resulting in a constant $M_{\mathrm{BH}}$ measurement, to within $\sim 0.05$ dex uncertainty 


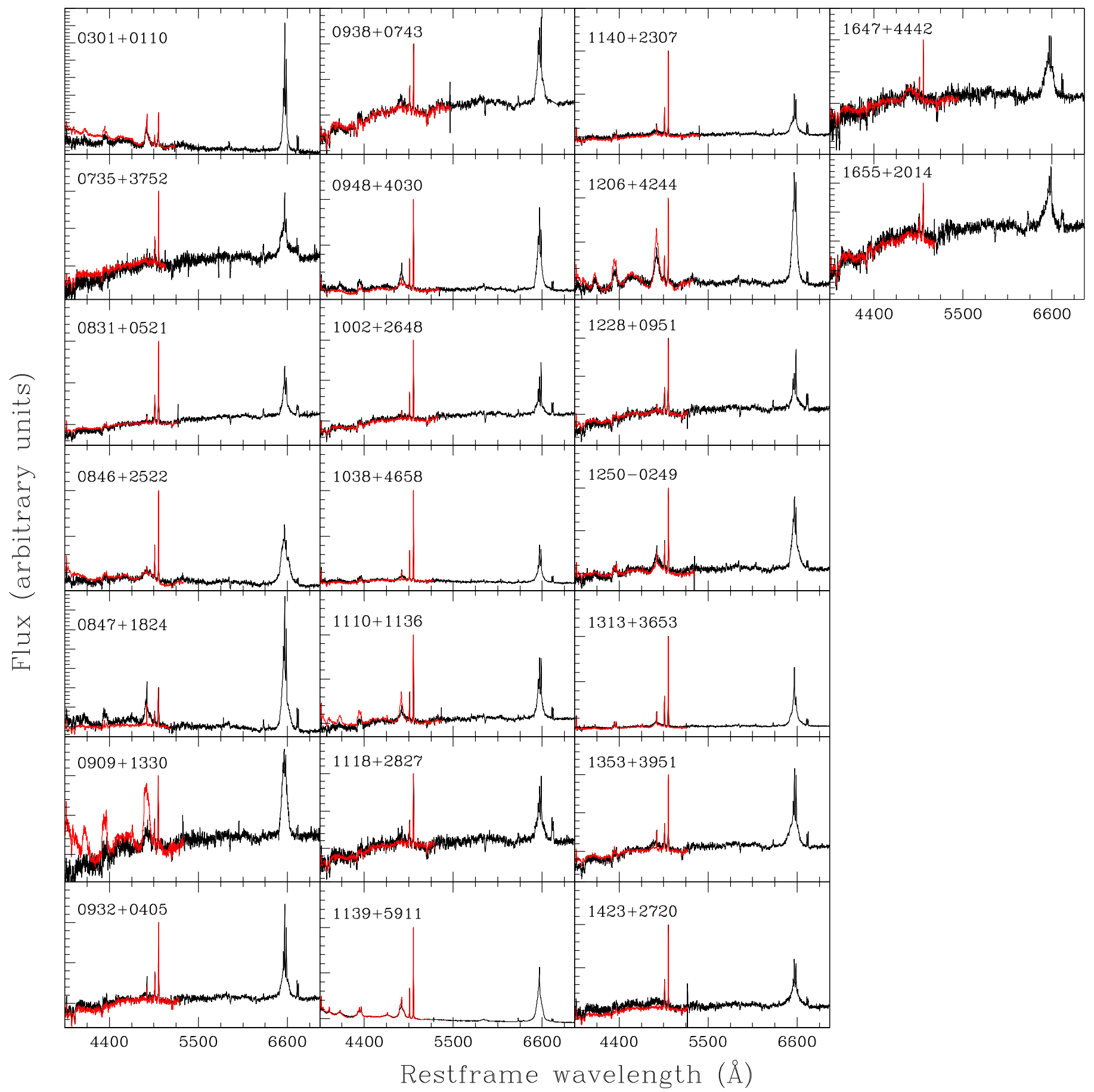

Figure 6. Same as Figure 3, except that these are the 23 objects not included in Bennert et al. (2015).

(see, e.g., Bentz et al. 2007; Park et al. 2012; Barth et al. 2015). Ruan et al. (2015) also find that for their changing look quasars, the decrease in luminosity coincides with a broadening of the line widths to preserve the derived $M_{\mathrm{BH}}$. Unfortunately we cannot test this correlation in this paper because we derive the host galaxy free continuum flux from a 2D image decomposition of the SDSS images (Bennert et al. 2015), taken at yet a different time from the two sets of spectra. We consider this approach superior over spectral decomposition, given unknown aperture effects for spectra. (For the same reason, image decomposition based on typically an Hubble Space Telescope image taken at a different time is also used for reverberation-mapped AGNs (e.g., Bentz et al. 2013).) Moreover, the Keck spectra are not absolutely flux calibrated due to typically non-photometric observing conditions. However, using the same continuum flux derived from the $2 \mathrm{D}$ image decomposition, but taking into account the different width of $\mathrm{H} \beta$ between the SDSS and Keck spectra, the resulting $M_{\mathrm{BH}}$ is on average $0.05 \pm 0.03$ dex larger for SDSS spectra. This is small compared with the uncertainty of single-epoch measurements of 0.4 dex. Note that this includes only the 79 objects from Bennert et al. 2015, since we do not have continuum measurement for the other objects. Also, for obvious reasons, 
Table 3

Results from Spectral Fitting

\begin{tabular}{|c|c|c|c|c|c|c|c|c|c|c|c|c|}
\hline Object & $\begin{array}{c}\sigma_{\mathrm{H} \beta} \\
\mathrm{SDSS} \\
\left(\mathrm{km} \mathrm{s}^{-1}\right) \\
(2)\end{array}$ & $\begin{array}{c}\mathrm{FWHM}_{\mathrm{H} \beta} \\
\mathrm{SDSS} \\
\left(\mathrm{km} \mathrm{s}^{-1}\right) \\
(3)\end{array}$ & $\begin{array}{c}\frac{\mathrm{H} \beta \text { narrow }}{\left[{ }_{\text {SDSI }} \text { Flux }\right.} \\
\text { (4) }\end{array}$ & $\begin{array}{c}\mathrm{H} \beta \frac{\text { broad }}{\text { narrow }} \text { Flux } \\
\text { SDSS } \\
\text { (5) }\end{array}$ & $\begin{array}{c}\mathrm{H} \beta \frac{\text { broad }}{\text { narrow }} \text { Peak Flux } \\
\text { SDSS } \\
\text { (6) }\end{array}$ & $\begin{array}{c}\text { Fe II } \\
\text { SDSS } \\
\text { (7) }\end{array}$ & $\begin{array}{c}\sigma_{\mathrm{H} \beta} \\
\mathrm{Keck} \\
\left(\mathrm{km} \mathrm{s}^{-1}\right) \\
(8)\end{array}$ & $\begin{array}{c}\mathrm{FWHM}_{\mathrm{H} \beta} \\
\mathrm{Keck} \\
\left(\mathrm{km} \mathrm{s}^{-1}\right) \\
(9)\end{array}$ & $\begin{array}{c}\frac{\mathrm{H} \beta \text { narrow }}{[\mathrm{O} \mathrm{III}]} \text { Flux } \\
\text { Keck } \\
(10)\end{array}$ & $\begin{array}{c}\mathrm{H} \beta \frac{\text { broad }}{\text { narrow }} \text { Flux } \\
\text { Keck } \\
\text { (11) }\end{array}$ & $\begin{array}{c}\mathrm{H} \beta \frac{\text { broad }}{\text { narrow }} \\
\text { Peak Flux } \\
\text { Keck } \\
\text { (12) }\end{array}$ & $\begin{array}{l}\mathrm{Fe} \text { II } \\
\text { Keck }\end{array}$ \\
\hline $0013-0951$ & $1783 \pm 135$ & $3462 \pm 205$ & $0.13 \pm 0.02$ & $65 \pm 10$ & $8 \pm 1$ & $\mathrm{Y}$ & $2111 \pm 211$ & $4275 \pm 594$ & $0.17 \pm 0.02$ & $20 \pm 2$ & $2.05 \pm 0.17$ & $\mathrm{Y}$ \\
\hline $0026+0009$ & $964 \pm 125$ & $2276 \pm 293$ & $0.18 \pm 0.05$ & $14 \pm 4$ & $2.7 \pm 0.7$ & $\mathrm{~N}$ & $1527 \pm 227$ & $2920 \pm 765$ & $0.26 \pm 0.06$ & $10 \pm 2$ & $1.4 \pm 0.3$ & $\mathrm{Y}$ \\
\hline $0038+0034$ & $2759 \pm 133$ & $7371 \pm 321$ & $0.08 \pm 0.01$ & $23.2 \pm 1.8$ & $1.54 \pm 0.09$ & $\mathrm{~N}$ & $3328 \pm 211$ & $7223 \pm 593$ & $0.09 \pm 0.01$ & $10 \pm 1$ & $0.63 \pm 0.04$ & $\mathrm{Y}$ \\
\hline $0109+0059$ & $1677 \pm 230$ & $3392 \pm 282$ & $0.15 \pm 0.02$ & $4.6 \pm 0.8$ & $0.70 \pm 0.09$ & $\mathrm{Y}$ & $1797 \pm 268$ & $3377 \pm 355$ & $0.11 \pm 0.01$ & $4.1 \pm 0.8$ & $0.61 \pm 0.06$ & $\mathrm{Y}$ \\
\hline $0121-0102$ & $2070 \pm 194$ & $4069 \pm 255$ & $0.15 \pm 0.03$ & $6 \pm 1$ & $0.9 \pm 0.2$ & $\mathrm{Y}$ & $1742 \pm 106$ & $3145 \pm 145$ & $0.10 \pm 0.01$ & $11 \pm 0.9$ & $1.67 \pm 0.09$ & $\mathrm{Y}$ \\
\hline $0150+0057$ & $1799 \pm 172$ & $4816 \pm 408$ & $0.09 \pm 0.01$ & $25 \pm 4$ & $2.0 \pm 0.2$ & $\mathrm{~N}$ & $2057 \pm 129$ & $4212 \pm 242$ & $0.07 \pm 0.01$ & $1 \pm 4$ & $3.7 \pm 0.3$ & $\mathrm{Y}$ \\
\hline $0206-0017$ & $2514 \pm 483$ & $5054 \pm 761$ & $0.12 \pm 0.03$ & $18 \pm 5$ & $1.68 \pm 0.18$ & $\mathrm{Y}$ & $1979 \pm 99$ & $4060 \pm 148$ & $0.10 \pm 0.01$ & $34 \pm 3$ & $4.7 \pm 0.3$ & $\mathrm{Y}$ \\
\hline $0212+1406$ & $1947 \pm 198$ & $3776 \pm 299$ & $0.11 \pm 0.01$ & $15.0 \pm 1.9$ & $1.6 \pm 0.1$ & $\mathrm{Y}$ & $1586 \pm 129$ & $2601 \pm 242$ & $0.15 \pm 0.01$ & $17.7 \pm 1.7$ & $2.6 \pm 0.2$ & $\mathrm{Y}$ \\
\hline $0301+0110$ & $2078 \pm 226$ & $3751 \pm 370$ & $0.47 \pm 0.07$ & $4.9 \pm 0.8$ & $0.90 \pm 0.08$ & $\mathrm{Y}$ & $1423 \pm 160$ & $3612 \pm 442$ & $0.42 \pm 0.06$ & $6.77 \pm 1.08$ & $1.16 \pm 0.11$ & $\mathrm{Y}$ \\
\hline $0301+0115$ & $1510 \pm 182$ & $3928 \pm 181$ & $0.05 \pm 0.01$ & $39 \pm 6$ & $6.2 \pm 0.5$ & $\mathrm{Y}$ & $1653 \pm 105$ & $3594 \pm 200$ & $0.09 \pm 0.01$ & $16.2 \pm 1.6$ & $2.73 \pm 0.17$ & $\mathrm{Y}$ \\
\hline $0310-0049$ & $1713 \pm 111$ & $3152 \pm 178$ & $0.09 \pm 0.01$ & $44 \pm 6$ & $2.9 \pm 0.4$ & $\mathrm{Y}$ & $1558 \pm 69$ & $2843 \pm 153$ & $0.08 \pm 0.02$ & $70 \pm 12$ & $4.9 \pm 0.8$ & $\mathrm{Y}$ \\
\hline $0336-0706$ & $3189 \pm 177$ & $6827 \pm 416$ & $0.19 \pm 0.02$ & $4.8 \pm 0.4$ & $0.32 \pm 0.02$ & $\mathrm{Y}$ & $2403 \pm 164$ & $7238 \pm 552$ & $0.16 \pm 0.04$ & $3.6 \pm 1.3$ & $0.24 \pm 0.06$ & $\mathrm{Y}$ \\
\hline $0353-0623$ & $1725 \pm 359$ & $4209 \pm 557$ & $0.12 \pm 0.03$ & $12 \pm 4$ & $1.0 \pm 0.2$ & $\mathrm{Y}$ & $1548 \pm 225$ & $3050 \pm 312$ & $0.13 \pm 0.01$ & $20 \pm 4$ & $2.12 \pm 0.15$ & $\mathrm{Y}$ \\
\hline $0731+4522$ & $1778 \pm 107$ & $3291 \pm 260$ & $0.18 \pm 0.01$ & $3.3 \pm 0.3$ & $0.53 \pm 0.03$ & $\mathrm{~N}$ & $1885 \pm 134$ & $3715 \pm 389$ & $0.16 \pm 0.01$ & $4.5 \pm 0.5$ & $0.72 \pm 0.04$ & $\mathrm{Y}$ \\
\hline $0735+3752$ & $3120 \pm 303$ & $4572 \pm 712$ & $0.18 \pm 0.06$ & $7 \pm 3$ & $0.37 \pm 0.13$ & $\mathrm{Y}$ & $3996 \pm 293$ & $8070 \pm 838$ & $0.14 \pm 0.06$ & $7 \pm 4$ & $0.30 \pm 0.12$ & $\mathrm{~N}$ \\
\hline $0737+4244$ & $1624 \pm 74$ & $4326 \pm 133$ & $0.22 \pm 0.01$ & $3.8 \pm 0.2$ & $0.41 \pm 0.02$ & $\mathrm{~N}$ & $1692 \pm 98$ & $3361 \pm 148$ & $0.19 \pm 0.01$ & $6.2 \pm 0.5$ & $0.82 \pm 0.05$ & $\mathrm{Y}$ \\
\hline $0802+3104$ & $2661 \pm 265$ & $5707 \pm 652$ & $0.10 \pm 0.02$ & $34 \pm 7$ & $2.0 \pm 0.4$ & $\mathrm{Y}$ & $1772 \pm 185$ & $4430 \pm 358$ & $0.07 \pm 0.01$ & $19.5 \pm 1.5$ & $1.66 \pm 0.11$ & $\mathrm{Y}$ \\
\hline $0811+1739$ & $1779 \pm 66$ & $4325 \pm 156$ & $0.06 \pm 0.01$ & $41 \pm 6$ & $2.4 \pm 0.3$ & $\mathrm{Y}$ & $1520 \pm 150$ & $3520 \pm 253$ & $0.11 \pm 0.02$ & $31 \pm 9$ & $2.6 \pm 0.5$ & $\mathrm{Y}$ \\
\hline $0813+4608$ & $1270 \pm 197$ & $2992 \pm 471$ & $0.08 \pm 0.08$ & $5 \pm 7$ & $0.3 \pm 0.3$ & $\mathrm{Y}$ & $1430 \pm 91$ & $2483 \pm 165$ & $0.11 \pm 0.01$ & $14.0 \pm 1.5$ & $1.39 \pm 0.11$ & $\mathrm{Y}$ \\
\hline $0831+0521$ & $1040 \pm 328$ & $1197 \pm 550$ & $0.10 \pm 0.02$ & $1.3 \pm 0.6$ & $0.32 \pm 0.08$ & $\mathrm{~N}$ & $\ldots$ & & $0.11 \pm 0.06$ & {$[0.06]$} & {$[0.32]$} & $\mathrm{N}$ \\
\hline $0845+3409$ & $2090 \pm 294$ & $3897 \pm 865$ & $0.16 \pm 0.02$ & $9.7 \pm 1.7$ & $0.69 \pm 0.07$ & $\mathrm{Y}$ & $1718 \pm 172$ & $2820 \pm 310$ & $0.16 \pm 0.02$ & $7.5 \pm 1.2$ & $0.69 \pm 0.08$ & $\mathrm{Y}$ \\
\hline $0846+2522$ & $2572 \pm 244$ & $7398 \pm 410$ & $0.09 \pm 0.02$ & $20 \pm 3$ & $1.04 \pm 0.14$ & $\mathrm{Y}$ & $3394 \pm 402$ & $8304 \pm 459$ & $0.08 \pm 0.02$ & $22 \pm 6$ & $0.97 \pm 0.15$ & $\mathrm{Y}$ \\
\hline $0847+1824$ & $1665 \pm 193$ & $3519 \pm 282$ & $0.40 \pm 0.04$ & $8.0 \pm 1.1$ & $1.19 \pm 0.08$ & $\mathrm{Y}$ & $\ldots$ & $\ldots$ & $0.27 \pm 0.11$ & [1.66] & {$[0.08]$} & $\mathrm{N}$ \\
\hline $0854+1741$ & $2729 \pm 353$ & $4642 \pm 602$ & $0.16 \pm 0.02$ & $5.67 \pm 1.08$ & $0.84 \pm 0.11$ & $\mathrm{Y}$ & $1472 \pm 269$ & $2582 \pm 563$ & $0.22 \pm 0.01$ & $5 \pm 1$ & $1.36 \pm 0.04$ & $\mathrm{Y}$ \\
\hline $0857+0528$ & $1959 \pm 193$ & $4307 \pm 282$ & $0.17 \pm 0.03$ & $22 \pm 4$ & $1.8 \pm 0.3$ & $\mathrm{Y}$ & $1485 \pm 120$ & $3499 \pm 51$ & $0.1 \pm 0.01$ & $17.0 \pm 1.3$ & $1.85 \pm 0.13$ & $\mathrm{Y}$ \\
\hline $0904+5536$ & $2643 \pm 70$ & $5724 \pm 125$ & $0.26 \pm 0.02$ & $7.8 \pm 0.4$ & $0.48 \pm 0.03$ & $\mathrm{Y}$ & $2483 \pm 36$ & $7540 \pm 182$ & $0.22 \pm 0.02$ & $7.5 \pm 0.5$ & $0.45 \pm 0.03$ & $\mathrm{Y}$ \\
\hline $0909+1330$ & $1721 \pm 251$ & $4023 \pm 416$ & $0.12 \pm 0.02$ & $18 \pm 4$ & $2.00 \pm 0.19$ & $\mathrm{Y}$ & $1687 \pm 138$ & $4418 \pm 148$ & $0.19 \pm 0.04$ & 45. \pm 8 & $4.4 \pm 0.5$ & $\mathrm{Y}$ \\
\hline $0921+1017$ & $2033 \pm 157$ & $4900 \pm 498$ & $0.23 \pm 0.04$ & $4.6 \pm 0.9$ & $0.31 \pm 0.05$ & Y & $2317 \pm 286$ & $4432 \pm 427$ & $0.17 \pm 0.02$ & $8.9 \pm 1.4$ & $0.71 \pm 0.06$ & $\mathrm{Y}$ \\
\hline $0923+2254$ & $2195 \pm 254$ & $3783 \pm 545$ & $0.51 \pm 0.13$ & $4.1 \pm 0.8$ & $0.67 \pm 0.15$ & $\mathrm{Y}$ & $1824 \pm 265$ & $2924 \pm 668$ & $0.24 \pm 0.03$ & $9.4 \pm 2.0$ & $1.66 \pm 0.19$ & $\mathrm{Y}$ \\
\hline $0923+2946$ & $2686 \pm 222$ & $5946 \pm 522$ & $0.13 \pm 0.02$ & $5.9 \pm 1.1$ & $0.24 \pm 0.03$ & $\mathrm{Y}$ & $2936 \pm 247$ & $5650 \pm 906$ & $0.16 \pm 0.02$ & $11.2 \pm 1.7$ & $0.56 \pm 0.06$ & $\mathrm{Y}$ \\
\hline $0927+2301$ & $2624 \pm 226$ & $7732 \pm 647$ & $0.10 \pm 0.03$ & $17 \pm 5$ & $1.0 \pm 0.2$ & $\mathrm{Y}$ & $2112 \pm 205$ & $5377 \pm 367$ & $0.08 \pm 0.01$ & $17 \pm 2$ & $1.5 \pm 0.1$ & $\mathrm{Y}$ \\
\hline $0932+0233$ & $2407 \pm 429$ & $6863 \pm 625$ & $0.13 \pm 0.01$ & $10 \pm 2$ & $0.50 \pm 0.04$ & $\mathrm{Y}$ & $1814 \pm 72$ & $4273 \pm 168$ & $0.13 \pm 0.01$ & $12.54 \pm 1.04$ & $1.05 \pm 0.07$ & $\mathrm{Y}$ \\
\hline $0932+0405$ & $1829 \pm 75$ & $5316 \pm 427$ & $0.26 \pm 0.02$ & $2.5 \pm 0.2$ & $0.24 \pm 0.02$ & $\mathrm{~N}$ & $\ldots$ & $\ldots$ & $0.1 \pm 0.4$ & {$[0.41]$} & {$[0]$} & $\mathrm{N}$ \\
\hline $0936+1014$ & $2259 \pm 153$ & $4846 \pm 228$ & $0.09 \pm 0.01$ & $9.9 \pm 0.8$ & $1.35 \pm 0.07$ & $\mathrm{Y}$ & $1995 \pm 80$ & $3916 \pm 123$ & $0.10 \pm 0.01$ & $17.02 \pm 1.07$ & $2.80 \pm 0.14$ & $\mathrm{Y}$ \\
\hline $0938+0743$ & $1663 \pm 190$ & $4813 \pm 684$ & $0.14 \pm 0.02$ & $11 \pm 2$ & $1.04 \pm 0.12$ & $\mathrm{~N}$ & $3723 \pm 608$ & $7110 \pm 808$ & $0.15 \pm 0.12$ & $4 \pm 4$ & $0.22 \pm 0.17$ & $\mathrm{~N}$ \\
\hline $0948+4030$ & $1768 \pm 225$ & $3460 \pm 258$ & $0.17 \pm 0.05$ & $12 \pm 4$ & $1.2 \pm 0.4$ & $\mathrm{Y}$ & $3188 \pm 438$ & $6732 \pm 548$ & $0.12 \pm 0.01$ & $11.0 \pm 1.8$ & $0.57 \pm 0.05$ & $\mathrm{Y}$ \\
\hline $1002+2648$ & $1944 \pm 184$ & $5721 \pm 433$ & $0.12 \pm 0.02$ & $4.6 \pm 1.0$ & $0.29 \pm 0.04$ & $\mathrm{Y}$ & $\cdots$ & & $0.12 \pm 0.04$ & {$[2.23]$} & {$[0.15]$} & $\mathrm{N}$ \\
\hline $1029+1408$ & $2031 \pm 338$ & $5264 \pm 381$ & $0.14 \pm 0.02$ & $9.8 \pm 2.0$ & $0.80 \pm 0.08$ & $\mathrm{Y}$ & $2456 \pm 344$ & $6499 \pm 649$ & $0.15 \pm 0.01$ & $8.7 \pm 1.5$ & $0.59 \pm 0.05$ & $\mathrm{Y}$ \\
\hline $1029+2728$ & $2103 \pm 233$ & $4958 \pm 547$ & $0.23 \pm 0.06$ & $4.1 \pm 1.5$ & $0.32 \pm 0.08$ & $\mathrm{Y}$ & $1544 \pm 28$ & $3634 \pm 152$ & $0.25 \pm 0.03$ & $5.07 \pm 1.06$ & $0.59 \pm 0.06$ & $\mathrm{Y}$ \\
\hline $1029+4019$ & $2515 \pm 349$ & $5985 \pm 401$ & $0.11 \pm 0.02$ & $8.4 \pm 1.5$ & $0.64 \pm 0.07$ & $\mathrm{Y}$ & $2193 \pm 387$ & $5998 \pm 547$ & $0.12 \pm 0.01$ & $6.5 \pm 1.3$ & $0.52 \pm 0.04$ & $\mathrm{Y}$ \\
\hline $1038+4658$ & $2621 \pm 322$ & $4750 \pm 302$ & $0.10 \pm 0.01$ & $9.4 \pm 1.4$ & $0.62 \pm 0.06$ & $\mathrm{Y}$ & $\ldots$ & $\ldots$ & $0.10 \pm 0.14$ & {$[2.75]$} & {$[0.14]$} & $\mathrm{N}$ \\
\hline $1042+0414$ & $1569 \pm 194$ & $4064 \pm 166$ & $0.34 \pm 0.03$ & $6.6 \pm 1.0$ & $0.57 \pm 0.04$ & $\mathrm{Y}$ & $1518 \pm 102$ & $2724 \pm 145$ & $0.33 \pm 0.03$ & $5.6 \pm 0.6$ & $0.73 \pm 0.05$ & $\mathrm{Y}$ \\
\hline $1043+1105$ & $2864 \pm 149$ & $6171 \pm 230$ & $0.21 \pm 0.04$ & $10.5 \pm 1.7$ & $0.61 \pm 0.06$ & $\mathrm{Y}$ & $2313 \pm 28$ & $6597 \pm 152$ & $0.13 \pm 0.01$ & $4.7 \pm 0.2$ & $0.25 \pm 0.02$ & $\mathrm{Y}$ \\
\hline $1049+2451$ & $2368 \pm 161$ & $5181 \pm 207$ & $0.15 \pm 0.03$ & $14.2 \pm 1.8$ & $0.97 \pm 0.14$ & $\mathrm{Y}$ & $2534 \pm 135$ & $5112 \pm 203$ & $0.15 \pm 0.01$ & $15.1 \pm 1.2$ & $1.04 \pm 0.07$ & $\mathrm{Y}$ \\
\hline
\end{tabular}


Table 3

(Continued)

\begin{tabular}{|c|c|c|c|c|c|c|c|c|c|c|c|c|}
\hline Object & $\begin{array}{c}\sigma_{\mathrm{H} \beta} \\
\mathrm{SDSS} \\
\left(\mathrm{km} \mathrm{s}^{-1}\right) \\
(2)\end{array}$ & $\begin{array}{l}\mathrm{FWHM}_{\mathrm{H} \beta} \\
\mathrm{SDSS} \\
\left(\mathrm{km} \mathrm{s}^{-1}\right) \\
(3)\end{array}$ & $\begin{array}{c}\frac{\mathrm{H} \beta \text { narrow }}{[0 \text { III] }} \text { Flux } \\
\text { SDSS } \\
\text { (4) }\end{array}$ & $\begin{array}{c}\mathrm{H} \beta \frac{\text { broad }}{\text { narrow }} \text { Flux } \\
\text { SDSS } \\
\text { (5) }\end{array}$ & $\begin{array}{c}\mathrm{H} \beta \frac{\text { broad }}{\text { narrow }} \text { Peak Flux } \\
\text { SDSS } \\
\text { (6) }\end{array}$ & $\begin{array}{l}\text { Fe II } \\
\text { SDSS } \\
(7)\end{array}$ & $\begin{array}{c}\sigma_{\mathrm{H} \beta} \\
\mathrm{Keck} \\
\left(\mathrm{km} \mathrm{s}^{-1}\right) \\
(8)\end{array}$ & $\begin{array}{l}\mathrm{FWHM}_{\mathrm{H} \beta} \\
\mathrm{Keck} \\
\left(\mathrm{km} \mathrm{s}^{-1}\right) \\
(9)\end{array}$ & $\begin{array}{c}\frac{\mathrm{H} \beta \text { narrow }}{[\mathrm{O} \text { III] }} \text { Flux } \\
\text { Keck } \\
(10)\end{array}$ & $\begin{array}{c}\mathrm{H} \beta \frac{\text { broad }}{\text { narrow }} \text { Flux } \\
\text { Keck } \\
\text { (11) }\end{array}$ & $\begin{array}{c}\mathrm{H} \beta \frac{\text { broad }}{\text { narrow }} \\
\text { Peak Flux } \\
\text { Keck } \\
(12)\end{array}$ & $\begin{array}{c}\mathrm{Fe} \text { II } \\
\text { Keck }\end{array}$ \\
\hline $1058+5259$ & $1987 \pm 400$ & $4928 \pm 940$ & $0.07 \pm 0.01$ & $18 \pm 5$ & $1.24 \pm 0.18$ & $\mathrm{~N}$ & $1896 \pm 150$ & $5372 \pm 150$ & $0.10 \pm 0.01$ & $13.3 \pm 1.7$ & $0.92 \pm 0.08$ & $\bar{Y}$ \\
\hline $1101+1102$ & $2558 \pm 125$ & $6047 \pm 295$ & $0.14 \pm 0.03$ & $7.6 \pm 1.2$ & $0.62 \pm 0.13$ & $\mathrm{~N}$ & $3949 \pm 170$ & $8349 \pm 597$ & $0.13 \pm 0.01$ & $3.5 \pm 0.3$ & $0.20 \pm 0.01$ & $\mathrm{Y}$ \\
\hline $1104+4334$ & $1873 \pm 308$ & $4319 \pm 647$ & $0.09 \pm 0.02$ & $4.4 \pm 1.3$ & $0.31 \pm 0.06$ & $\mathrm{Y}$ & $1719 \pm 160$ & $4072 \pm 395$ & $0.12 \pm 0.01$ & $9.4 \pm 1.2$ & $0.84 \pm 0.07$ & $\mathrm{Y}$ \\
\hline $1110+1136$ & $1878 \pm 206$ & $3860 \pm 400$ & $0.14 \pm 0.01$ & $10.4 \pm 1.6$ & $0.78 \pm 0.05$ & $\mathrm{Y}$ & $1378 \pm 95$ & $2898 \pm 150$ & $0.15 \pm 0.01$ & $14.7 \pm 1.5$ & $1.68 \pm 0.12$ & $\mathrm{Y}$ \\
\hline $1116+4123$ & $2531 \pm 294$ & $6324 \pm 692$ & $0.25 \pm 0.04$ & $10.2 \pm 1.9$ & $0.56 \pm 0.07$ & $\mathrm{~N}$ & $3136 \pm 315$ & $6774 \pm 740$ & $0.27 \pm 0.04$ & $6.6 \pm 1.3$ & $0.39 \pm 0.05$ & $\mathrm{Y}$ \\
\hline $1118+2827$ & $1908 \pm 136$ & $5910 \pm 498$ & $0.21 \pm 0.03$ & $3.8 \pm 0.7$ & $0.38 \pm 0.05$ & $\mathrm{~N}$ & & $\ldots$ & $0.14 \pm 0.05$ & {$[2.05]$} & {$[0.19]$} & $\mathrm{N}$ \\
\hline $1132+1017$ & $2028 \pm 147$ & $5782 \pm 345$ & $0.10 \pm 0.01$ & $7.7 \pm 1.3$ & $0.74 \pm 0.09$ & $\mathrm{Y}$ & $1900 \pm 86$ & $5341 \pm 740$ & $0.09 \pm 0.01$ & $15.8 \pm 1.4$ & $1.63 \pm 0.11$ & $\mathrm{Y}$ \\
\hline $1137+4826$ & $1750 \pm 357$ & $3663 \pm 647$ & $0.34 \pm 0.05$ & $5.8 \pm 1.4$ & $0.94 \pm 0.14$ & Y & $1606 \pm 92$ & $3788 \pm 222$ & $0.45 \pm 0.06$ & $6.8 \pm 0.9$ & $1.00 \pm 0.09$ & $\mathrm{Y}$ \\
\hline $1139+5911$ & $2333 \pm 158$ & $4262 \pm 218$ & $0.16 \pm 0.06$ & $20 \pm 4$ & $2.2 \pm 0.7$ & $\mathrm{Y}$ & $2228 \pm 111$ & $3994 \pm 221$ & $0.09 \pm 0.01$ & $22.2 \pm 1.7$ & $1.73 \pm 0.11$ & $\mathrm{Y}$ \\
\hline $1140+2307$ & $2710 \pm 235$ & $5014 \pm 668$ & $0.10 \pm 0.01$ & $8.0 \pm 1.2$ & $0.43 \pm 0.05$ & $\mathrm{~N}$ & $3324 \pm 330$ & $6376 \pm 586$ & $0.12 \pm 0.01$ & $4.4 \pm 0.6$ & $0.24 \pm 0.02$ & $\mathrm{~N}$ \\
\hline $1143+5941$ & $2002 \pm 446$ & $5629 \pm 863$ & $0.13 \pm 0.04$ & $15.0 \pm 2.7$ & $0.77 \pm 0.18$ & $\mathrm{Y}$ & $1790 \pm 128$ & $5405 \pm 424$ & $0.08 \pm 0.01$ & $38 \pm 4$ & $2.00 \pm 0.08$ & $\mathrm{Y}$ \\
\hline $1144+3653$ & $3016 \pm 292$ & $8301 \pm 579$ & $0.08 \pm 0.01$ & $21 \pm 3$ & $0.90 \pm 0.14$ & $\mathrm{~N}$ & $2933 \pm 205$ & $8009 \pm 145$ & $0.08 \pm 0.01$ & $16.3 \pm 1.5$ & $0.87 \pm 0.05$ & $\mathrm{~N}$ \\
\hline $1145+5547$ & $2078 \pm 422$ & $4298 \pm 611$ & $0.10 \pm 0.01$ & $14 \pm 3$ & $1.18 \pm 0.12$ & $\mathrm{Y}$ & $1837 \pm 208$ & $4465 \pm 391$ & $0.15 \pm 0.01$ & $7.49 \pm 1.06$ & $0.71 \pm 0.05$ & $\mathrm{Y}$ \\
\hline $1147+0902$ & $3733 \pm 226$ & $6475 \pm 563$ & $0.14 \pm 0.05$ & $13 \pm 3$ & $0.9 \pm 0.3$ & $\mathrm{~N}$ & $2896 \pm 188$ & $5285 \pm 543$ & $0.12 \pm 0.01$ & $10.9 \pm 1.2$ & $0.73 \pm 0.06$ & $\mathrm{Y}$ \\
\hline $1205+4959$ & $3572 \pm 201$ & $8275 \pm 650$ & $0.11 \pm 0.02$ & $3.9 \pm 0.4$ & $0.24 \pm 0.03$ & $\mathrm{Y}$ & $2678 \pm 294$ & $5552 \pm 374$ & $0.10 \pm 0.01$ & $3.6 \pm 0.5$ & $0.31 \pm 0.02$ & $\mathrm{Y}$ \\
\hline $1206+4244$ & $1925 \pm 167$ & $3889 \pm 211$ & $0.15 \pm 0.02$ & $24 \pm 3$ & $2.4 \pm 0.3$ & Y & $1614 \pm 92$ & $3800 \pm 144$ & $0.17 \pm 0.02$ & $36 \pm 3$ & $3.6 \pm 0.3$ & $\mathrm{Y}$ \\
\hline $1210+3820$ & $2499 \pm 432$ & $6413 \pm 513$ & $0.20 \pm 0.04$ & $5.9 \pm 1.5$ & $0.36 \pm 0.06$ & $\mathrm{~N}$ & $2831 \pm 148$ & $5300 \pm 392$ & $0.20 \pm 0.02$ & $8.0 \pm 0.7$ & $0.58 \pm 0.04$ & $\mathrm{Y}$ \\
\hline $1216+5049$ & $3329 \pm 180$ & $8923 \pm 422$ & $0.11 \pm 0.02$ & $2.3 \pm 0.3$ & $0.19 \pm 0.03$ & $\mathrm{Y}$ & $4487 \pm 477$ & $7810 \pm 392$ & $0.09 \pm 0.01$ & $2.1 \pm 0.3$ & $0.15 \pm 0.01$ & $\mathrm{Y}$ \\
\hline $1223+0240$ & $2780 \pm 160$ & $5802 \pm 220$ & $0.05 \pm 0.02$ & $207 \pm 44$ & $17 \pm 6$ & $\mathrm{Y}$ & $2306 \pm 107$ & $5051 \pm 168$ & $0.10 \pm 0.01$ & $69 \pm 6$ & $5.7 \pm 0.4$ & $\mathrm{Y}$ \\
\hline $1228+0951$ & $2289 \pm 657$ & $7303 \pm 1543$ & $0.12 \pm 0.07$ & $3 \pm 3$ & $0.23 \pm 0.14$ & Y & $2331 \pm 456$ & $6011 \pm 495$ & $0.12 \pm 0.04$ & $3.2 \pm 1.6$ & $0.28 \pm 0.10$ & $\mathrm{Y}$ \\
\hline $1231+4504$ & $1551 \pm 343$ & $2872 \pm 440$ & $0.20 \pm 0.03$ & $5.5 \pm 1.4$ & $1.27 \pm 0.14$ & Y & $1555 \pm 168$ & $3325 \pm 394$ & $0.16 \pm 0.02$ & $9.1 \pm 1.2$ & $1.91 \pm 0.16$ & $\mathrm{Y}$ \\
\hline $1241+3722$ & $1829 \pm 93$ & $4320 \pm 219$ & $0.13 \pm 0.02$ & $7.9 \pm 0.9$ & $0.69 \pm 0.08$ & $\mathrm{~N}$ & $1574 \pm 100$ & $3185 \pm 197$ & $0.11 \pm 0.01$ & $6.0 \pm 0.8$ & $0.70 \pm 0.07$ & $\mathrm{Y}$ \\
\hline $1246+5134$ & $2402 \pm 313$ & $4403 \pm 703$ & $0.07 \pm 0.05$ & $10 \pm 11$ & $0.6 \pm 0.5$ & $\mathrm{Y}$ & $1141 \pm 130$ & $2270 \pm 185$ & $0.09 \pm 0.01$ & $12.0 \pm 1.8$ & $1.52 \pm 0.12$ & $\mathrm{Y}$ \\
\hline $1250-0249$ & $2068 \pm 323$ & $5304 \pm 732$ & $0.17 \pm 0.03$ & $9 \pm 2$ & $0.89 \pm 0.13$ & $\mathrm{Y}$ & $2417 \pm 246$ & $5771 \pm 541$ & $0.18 \pm 0.01$ & $7.3 \pm 0.9$ & $0.61 \pm 0.04$ & $\mathrm{Y}$ \\
\hline $1306+4552$ & $1327 \pm 148$ & $3262 \pm 237$ & $0.15 \pm 0.02$ & $18 \pm 3$ & $1.9 \pm 0.2$ & $\mathrm{~N}$ & $1892 \pm 297$ & $4129 \pm 772$ & $0.22 \pm 0.04$ & $2.8 \pm 0.8$ & $0.27 \pm 0.05$ & $\mathrm{Y}$ \\
\hline $1307+0952$ & $1616 \pm 114$ & $3748 \pm 267$ & $0.09 \pm 0.04$ & $11 \pm 7$ & $0.8 \pm 0.3$ & $\mathrm{~N}$ & $1630 \pm 165$ & $3586 \pm 249$ & $0.14 \pm 0.01$ & $12.7 \pm 1.7$ & $1.10 \pm 0.09$ & $\mathrm{~N}$ \\
\hline $1312+2628$ & $1585 \pm 171$ & $3131 \pm 256$ & $0.12 \pm 0.03$ & $32 \pm 10$ & $2.8 \pm 0.5$ & $\mathrm{Y}$ & $1572 \pm 150$ & $2924 \pm 345$ & $0.22 \pm 0.03$ & $36 \pm 6$ & $2.95 \pm 0.17$ & $\mathrm{Y}$ \\
\hline $1313+3653$ & $2592 \pm 217$ & $5591 \pm 402$ & $0.14 \pm 0.02$ & $4.8 \pm 0.5$ & $0.34 \pm 0.03$ & $\mathrm{Y}$ & $2115 \pm 264$ & $4920 \pm 347$ & $0.13 \pm 0.01$ & $4.2 \pm 0.6$ & $0.34 \pm 0.02$ & $\mathrm{Y}$ \\
\hline $1323+2701$ & $4266 \pm 349$ & $10123 \pm 299$ & $0.089 \pm 0.004$ & $18.3 \pm 1.7$ & $0.79 \pm 0.02$ & $\mathrm{Y}$ & $2414 \pm 376$ & $5219 \pm 782$ & $0.10 \pm 0.01$ & $5.9 \pm 1.2$ & $0.54 \pm 0.06$ & $\mathrm{Y}$ \\
\hline $1353+3951$ & $2037 \pm 121$ & $6308 \pm 537$ & $0.19 \pm 0.02$ & $4.1 \pm 0.5$ & $0.31 \pm 0.02$ & $\mathrm{~N}$ & $\ldots$ & $\ldots$ & $0.2 \pm 0.7$ & {$[1.52]$} & {$[0.31]$} & $\mathrm{N}$ \\
\hline $1355+3834$ & $2842 \pm 79$ & $5936 \pm 245$ & $0.28 \pm 0.05$ & $3.3 \pm 0.3$ & $0.36 \pm 0.06$ & $\mathrm{Y}$ & $4034 \pm 301$ & $6371 \pm 277$ & $0.20 \pm 0.02$ & $3.2 \pm 0.3$ & $0.22 \pm 0.01$ & $\mathrm{~N}$ \\
\hline $1405-0259$ & $1873 \pm 212$ & $3518 \pm 549$ & $0.21 \pm 0.02$ & $13 \pm 2$ & $1.48 \pm 0.13$ & $\mathrm{Y}$ & $1599 \pm 140$ & $2933 \pm 260$ & $0.16 \pm 0.02$ & $11.1 \pm 1.4$ & $1.48 \pm 0.12$ & $\mathrm{Y}$ \\
\hline $1416+0317$ & $11853 \pm 251$ & $4022 \pm 589$ & $0.12 \pm 0.02$ & $5.4 \pm 1.1$ & $0.78 \pm 0.09$ & $\mathrm{Y}$ & $1514 \pm 233$ & $3565 \pm 550$ & $0.11 \pm 0.02$ & $2.2 \pm 0.5$ & $0.40 \pm 0.05$ & $\mathrm{Y}$ \\
\hline $1419+0754$ & $1940 \pm 154$ & $5517 \pm 362$ & $0.10 \pm 0.02$ & $2.9 \pm 0.7$ & $0.35 \pm 0.06$ & $\mathrm{~N}$ & $3006 \pm 371$ & $5780 \pm 529$ & $0.10 \pm 0.02$ & $3.5 \pm 1.0$ & $0.36 \pm 0.07$ & $\mathrm{Y}$ \\
\hline $1423+2720$ & $3428 \pm 264$ & $7873 \pm 621$ & $0.11 \pm 0.03$ & $12 \pm 4$ & $0.5 \pm 0.1$ & $\mathrm{Y}$ & $\ldots$ & $\ldots$ & $0.08 \pm 0.04$ & {$[2.45]$} & {$[0]$} & $\mathrm{Y}$ \\
\hline $1434+4839$ & $2268 \pm 171$ & $4351 \pm 534$ & $0.08 \pm 0.02$ & $35 \pm 7$ & $2.7 \pm 0.6$ & $\mathrm{Y}$ & $1731 \pm 85$ & $4475 \pm 222$ & $0.09 \pm 0.01$ & $6.0 \pm 0.4$ & $0.58 \pm 0.03$ & $\mathrm{Y}$ \\
\hline $1505+0342$ & $2280 \pm 215$ & $5028 \pm 569$ & $0.14 \pm 0.03$ & $6.9 \pm 1.3$ & $0.83 \pm 0.12$ & $\mathrm{~N}$ & $1956 \pm 139$ & $5782 \pm 154$ & $0.08 \pm 0.01$ & $17.8 \pm 1.6$ & $1.55 \pm 0.09$ & $\mathrm{Y}$ \\
\hline $1535+5754$ & $2431 \pm 311$ & $4191 \pm 565$ & $0.09 \pm 0.03$ & $16 \pm 5$ & $1.4 \pm 0.4$ & $\mathrm{Y}$ & $2442 \pm 93$ & $5088 \pm 127$ & $0.08 \pm 0.01$ & $16.2 \pm 1.3$ & $1.41 \pm 0.07$ & $\mathrm{Y}$ \\
\hline $1543+3631$ & $1527 \pm 171$ & $2849 \pm 197$ & $0.14 \pm 0.02$ & $7.1 \pm 1.1$ & $1.03 \pm 0.15$ & $\mathrm{Y}$ & $1820 \pm 168$ & $3831 \pm 248$ & $0.09 \pm 0.01$ & $4.0 \pm 0.5$ & $0.47 \pm 0.03$ & $\mathrm{Y}$ \\
\hline $1545+1709$ & $2158 \pm 156$ & $5555 \pm 275$ & $0.09 \pm 0.01$ & $6.5 \pm 0.6$ & $0.52 \pm 0.05$ & $\mathrm{~N}$ & $3588 \pm 226$ & $4612 \pm 237$ & $0.06 \pm 0.01$ & $24 \pm 3$ & $1.68 \pm 0.14$ & $\mathrm{~N}$ \\
\hline $1554+3238$ & $2067 \pm 104$ & $4887 \pm 246$ & $0.14 \pm 0.02$ & $7.2 \pm 0.8$ & $0.83 \pm 0.11$ & $\mathrm{~N}$ & $2523 \pm 159$ & $4148 \pm 258$ & $0.11 \pm 0.01$ & $7.9 \pm 0.7$ & $1.04 \pm 0.06$ & $\mathrm{Y}$ \\
\hline $1557+0830$ & $13174 \pm 214$ & $5054 \pm 185$ & $0.18 \pm 0.03$ & $11.9 \pm 1.5$ & $0.87 \pm 0.09$ & $\mathrm{Y}$ & $2388 \pm 91$ & $4817 \pm 156$ & $0.17 \pm 0.02$ & $15.1 \pm 1.4$ & $1.09 \pm 0.08$ & $\mathrm{Y}$ \\
\hline $1605+3305$ & $2153 \pm 101$ & $5079 \pm 230$ & $0.06 \pm 0.02$ & $54 \pm 13$ & $2.9 \pm 1.0$ & $\mathrm{Y}$ & $1960 \pm 272$ & $5302 \pm 637$ & $0.10 \pm 0.01$ & $33 \pm 5$ & $2.03 \pm 0.14$ & $\mathrm{Y}$ \\
\hline $1606+3324$ & $2158 \pm 170$ & $5087 \pm 393$ & $0.10 \pm 0.01$ & $3.8 \pm 0.5$ & $0.34 \pm 0.04$ & $\mathrm{~N}$ & $2053 \pm 80$ & $5088 \pm 739$ & $0.12 \pm 0.01$ & $4.4 \pm 0.3$ & $0.45 \pm 0.03$ & $\mathrm{Y}$ \\
\hline
\end{tabular}


Table 3

(Continued)

\begin{tabular}{|c|c|c|c|c|c|c|c|c|c|c|c|c|}
\hline Object & $\begin{array}{c}\sigma_{\mathrm{H} \beta} \\
\mathrm{SDSS}^{-1} \\
\left(\mathrm{~km} \mathrm{~s}^{-1}\right) \\
(2)\end{array}$ & $\begin{array}{l}\mathrm{FWHM}_{\mathrm{H} \beta} \\
\mathrm{SDSS}^{-1} \\
\left(\mathrm{~km} \mathrm{~s}^{-1}\right) \\
(3)\end{array}$ & $\begin{array}{l}\frac{\mathrm{H} \beta \text { narrow }}{\left[{ }^{\circ} \text { III] }\right.} \text { Flux } \\
\text { (4) }\end{array}$ & $\begin{array}{c}\mathrm{H} \beta \frac{\text { broad }}{\text { narrow }} \text { Flux } \\
\text { SDSS } \\
\text { (5) }\end{array}$ & $\begin{array}{c}\mathrm{H} \beta \frac{\text { broad }}{\text { narrow }} \text { Peak Flux } \\
\text { SDSS } \\
\text { (6) }\end{array}$ & $\begin{array}{l}\mathrm{Fe} \text { II } \\
\text { SDSS } \\
\\
(7)\end{array}$ & $\begin{array}{c}\sigma_{\mathrm{H} \beta} \\
\mathrm{Keck} \\
\left(\mathrm{km} \mathrm{s}^{-1}\right) \\
(8)\end{array}$ & $\begin{array}{c}\mathrm{FWHM}_{\mathrm{H} \beta} \\
\mathrm{Keck} \\
\left(\mathrm{km} \mathrm{s}^{-1}\right) \\
(9)\end{array}$ & $\begin{array}{c}\frac{\mathrm{H} \beta \text { narrow }}{[\mathrm{O} \text { III] }} \text { Flux } \\
\text { Keck } \\
\text { (10) }\end{array}$ & $\begin{array}{c}\mathrm{H} \beta \frac{\text { broad }}{\text { narrow }} \text { Flux } \\
\text { Keck } \\
\text { (11) }\end{array}$ & $\begin{array}{c}\mathrm{H} / \delta \overline{\text { narrow }} \\
\text { Peak Flux } \\
\text { Keck } \\
(12)\end{array}$ & $\begin{array}{c}\mathrm{Fe} \text { II } \\
\text { Keck }\end{array}$ \\
\hline $1611+5211$ & $1392 \pm 207$ & $3895 \pm 486$ & $0.16 \pm 0.03$ & $4.8 \pm 1.1$ & $0.71 \pm 0.10$ & $\mathrm{~N}$ & $2515 \pm 410$ & $7695 \pm 964$ & $0.12 \pm 0.03$ & $2.9 \pm 1.1$ & $0.27 \pm 0.07$ & $\mathrm{Y}$ \\
\hline $1636+4202$ & $2367 \pm 223$ & $6655 \pm 621$ & $0.07 \pm 0.01$ & $39 \pm 7$ & $3.7 \pm 0.4$ & Y & $2492 \pm 230$ & $4542 \pm 523$ & $0.10 \pm 0.01$ & $24 \pm 3$ & $2.45 \pm 0.19$ & Y \\
\hline $1647+4442$ & $2227 \pm 279$ & $6228 \pm 655$ & $0.09 \pm 0.02$ & $4.8 \pm 1.9$ & $1.3 \pm 0.4$ & $\mathrm{~N}$ & $2921 \pm 246$ & $8325 \pm 214$ & $0.20 \pm 0.03$ & $21 \pm 4$ & $0.77 \pm 0.08$ & $\mathrm{~N}$ \\
\hline $1655+2014$ & $\ldots$ & $\ldots$ & $\ldots$ & $\ldots$ & $\ldots$ & $\mathrm{N}$ & $\ldots$ & $\ldots$ & $\ldots$ & $\ldots$ & $\ldots$ & $\mathrm{N}$ \\
\hline $1708+2153$ & $2829 \pm 134$ & $6055 \pm 580$ & $0.11 \pm 0.01$ & $15.2 \pm 1.2$ & $1.49 \pm 0.08$ & Y & $2402 \pm 122$ & $7359 \pm 245$ & $0.13 \pm 0.02$ & $62 \pm 10$ & $6.2 \pm 0.6$ & $\mathrm{Y}$ \\
\hline $2116+1102$ & $2790 \pm 27$ & $6577 \pm 64$ & $0.0903 \pm 0.0002$ & $2.25 \pm 0.02$ & $0.1831 \pm 0.0003$ & Y & $2484 \pm 42$ & $7186 \pm 248$ & $0.084 \pm 0.005$ & $3.63 \pm 0.18$ & $0.33 \pm 0.01$ & $\mathrm{Y}$ \\
\hline $2140+0025$ & $1329 \pm 104$ & $2225 \pm 127$ & $0.53 \pm 0.09$ & $7.1 \pm 1.1$ & $1.47 \pm 0.12$ & Y & $1114 \pm 64$ & $2155 \pm 127$ & $0.29 \pm 0.04$ & $17 \pm 2$ & $3.7 \pm 0.4$ & Y \\
\hline $2215-0036$ & $1877 \pm 200$ & $3330 \pm 155$ & $0.12 \pm 0.01$ & $8.5 \pm 1.1$ & $1.58 \pm 0.14$ & $\mathrm{Y}$ & $1636 \pm 92$ & $3966 \pm 220$ & $0.09 \pm 0.01$ & $7.3 \pm 0.6$ & $1.14 \pm 0.06$ & $\mathrm{Y}$ \\
\hline $2221-0906$ & $2498 \pm 394$ & $6684 \pm 539$ & $0.10 \pm 0.01$ & $21 \pm 4$ & $1.20 \pm 0.10$ & $\mathrm{~N}$ & $2375 \pm 131$ & $6012 \pm 224$ & $0.12 \pm 0.01$ & $24 \pm 2$ & $1.45 \pm 0.11$ & $\mathrm{Y}$ \\
\hline $2222-0819$ & $1811 \pm 88$ & $3327 \pm 319$ & $0.25 \pm 0.02$ & $2.5 \pm 0.2$ & $0.55 \pm 0.03$ & $\mathrm{~N}$ & $1799 \pm 168$ & $2861 \pm 343$ & $0.2 \pm 0.02$ & $2.5 \pm 0.3$ & $0.66 \pm 0.03$ & Y \\
\hline $2233+1312$ & $1897 \pm 66$ & $4409 \pm 236$ & $0.19 \pm 0.02$ & $6.7 \pm 0.4$ & $0.90 \pm 0.06$ & $\mathrm{~N}$ & $2477 \pm 135$ & $5830 \pm 318$ & $0.16 \pm 0.01$ & $5.7 \pm 0.5$ & $0.53 \pm 0.04$ & $\mathrm{~N}$ \\
\hline $2254+0046$ & $1466 \pm 200$ & $2015 \pm 195$ & $0.56 \pm 0.09$ & $3.9 \pm 0.8$ & $1.26 \pm 0.17$ & Y & $859 \pm 194$ & $1398 \pm 286$ & $0.59 \pm 0.07$ & $4.7 \pm 1.1$ & $1.66 \pm 0.18$ & Y \\
\hline $2327+1524$ & $3267 \pm 206$ & $4098 \pm 707$ & $0.06 \pm 0.01$ & $11.0 \pm 1.6$ & $1.10 \pm 0.13$ & $\mathrm{~N}$ & $1924 \pm 166$ & $5807 \pm 390$ & $0.08 \pm 0.02$ & $2.0 \pm 0.7$ & $0.22 \pm 0.06$ & $\mathrm{~N}$ \\
\hline $2351+1552$ & $3533 \pm 269$ & $10437 \pm 526$ & $0.12 \pm 0.01$ & $3.6 \pm 0.4$ & $0.20 \pm 0.02$ & $\mathrm{~N}$ & $2974 \pm 144$ & $7803 \pm 394$ & $0.11 \pm 0.01$ & $6.7 \pm 0.6$ & $0.43 \pm 0.03$ & $\mathrm{~N}$ \\
\hline
\end{tabular}

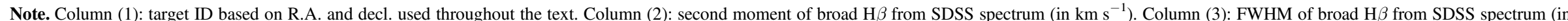

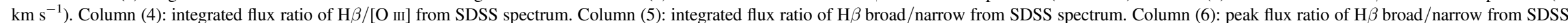

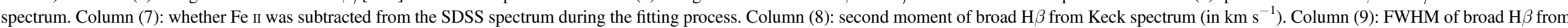

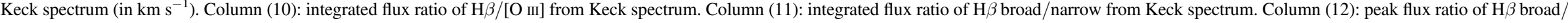

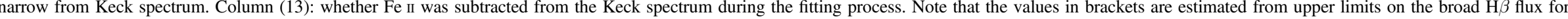

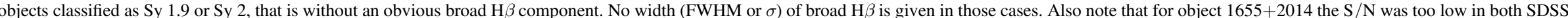
and Keck spectra to produce a reliable fit to the $\mathrm{H} \beta$ region. No values are given for this object and it is not included in the quantitative analysis. 


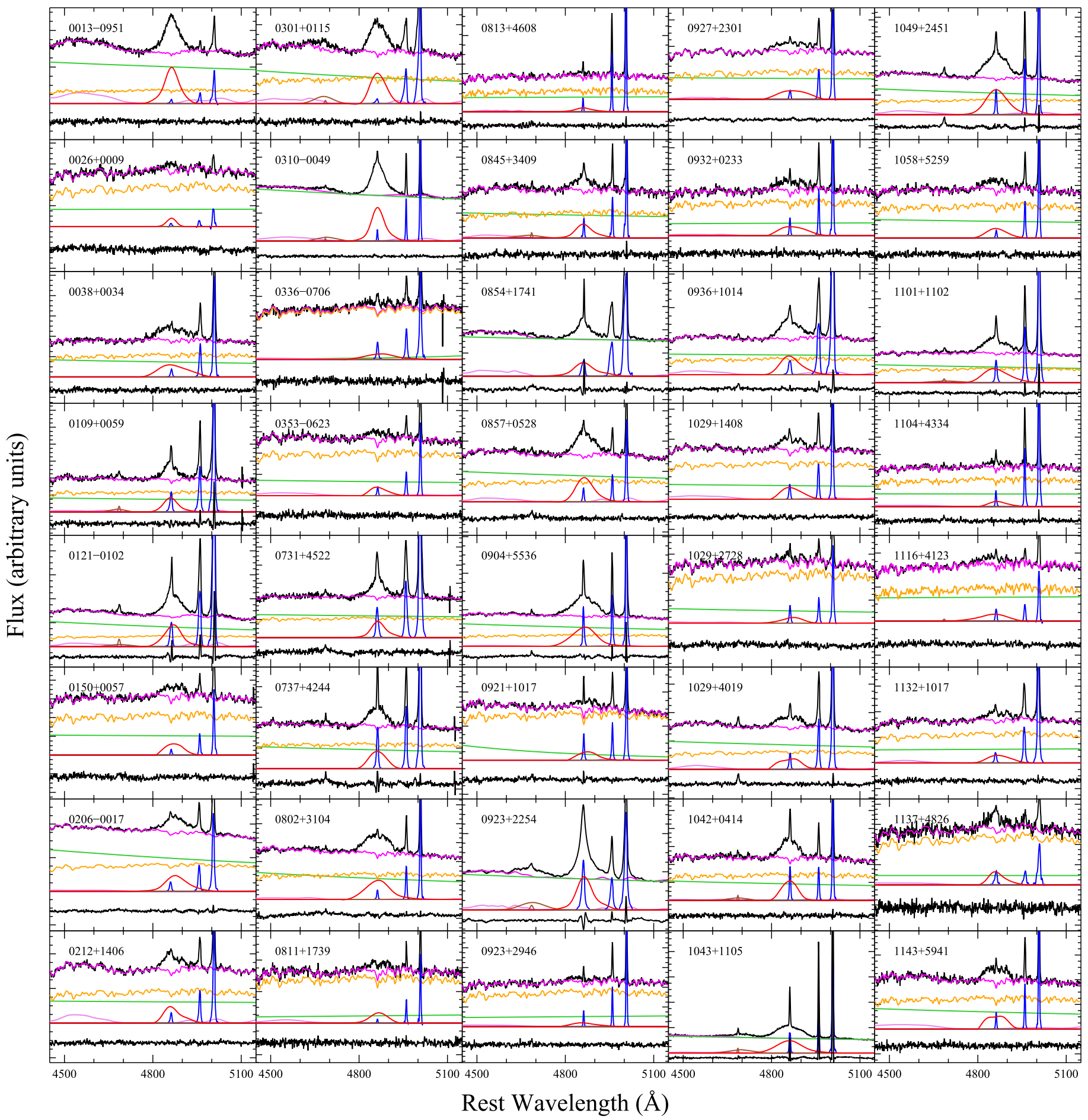

Figure 7. Multi-component spectral decomposition of the $\mathrm{H} \beta$ region in SDSS spectra. In the upper region, the observed spectrum is shown in black with the best fit of the continuum, Fe II, and host galaxy starlight in magenta. Below that the best fit to the power-law continuum is shown in green with the stellar spectrum in yellow. Below this the narrow lines of $\mathrm{H} \beta \lambda 4861$, and [O III] $\lambda \lambda 4959,5007$ are shown in blue, the broad and narrow components of He II $\lambda 4686$ in brown, and the broad component of $\mathrm{H} \beta$ in red. The residuals are plotted in black (arbitrarily shifted downward for clarity). Note that the objects shown here are included in Bennert et al. (2015), who show the corresponding fit to the Keck spectra in their Figure 3.

it excludes any object without broad $\mathrm{H} \beta$ emission in the Keck spectra.

The lower left panel of Figure 2 shows that while overall the $\mathrm{H} \beta_{\text {narrow }} /[\mathrm{O}$ III] flux ratio as measured in the SDSS spectra is comparable to that in the Keck spectra (on average $1.04 \pm 0.32$ ) with the majority of the objects falling near the unity line, the scatter is large due to some extreme outliers. While the large scatter could partially be due to the change in broad $\mathrm{H} \beta$ (as a consequence of the observed Seyfert-type change) effecting the narrow $\mathrm{H} \beta$ flux for fitting reasons since the lines are blended, we consider such an effect negligible given the quality of our data and our fitting procedure. More likely it might indicate that the NLR emission lines are not constant over the timescales of these observations but do indeed reverberate, which is in line with recent studies by (Peterson et al. 2013; Barth et al. 2015). 


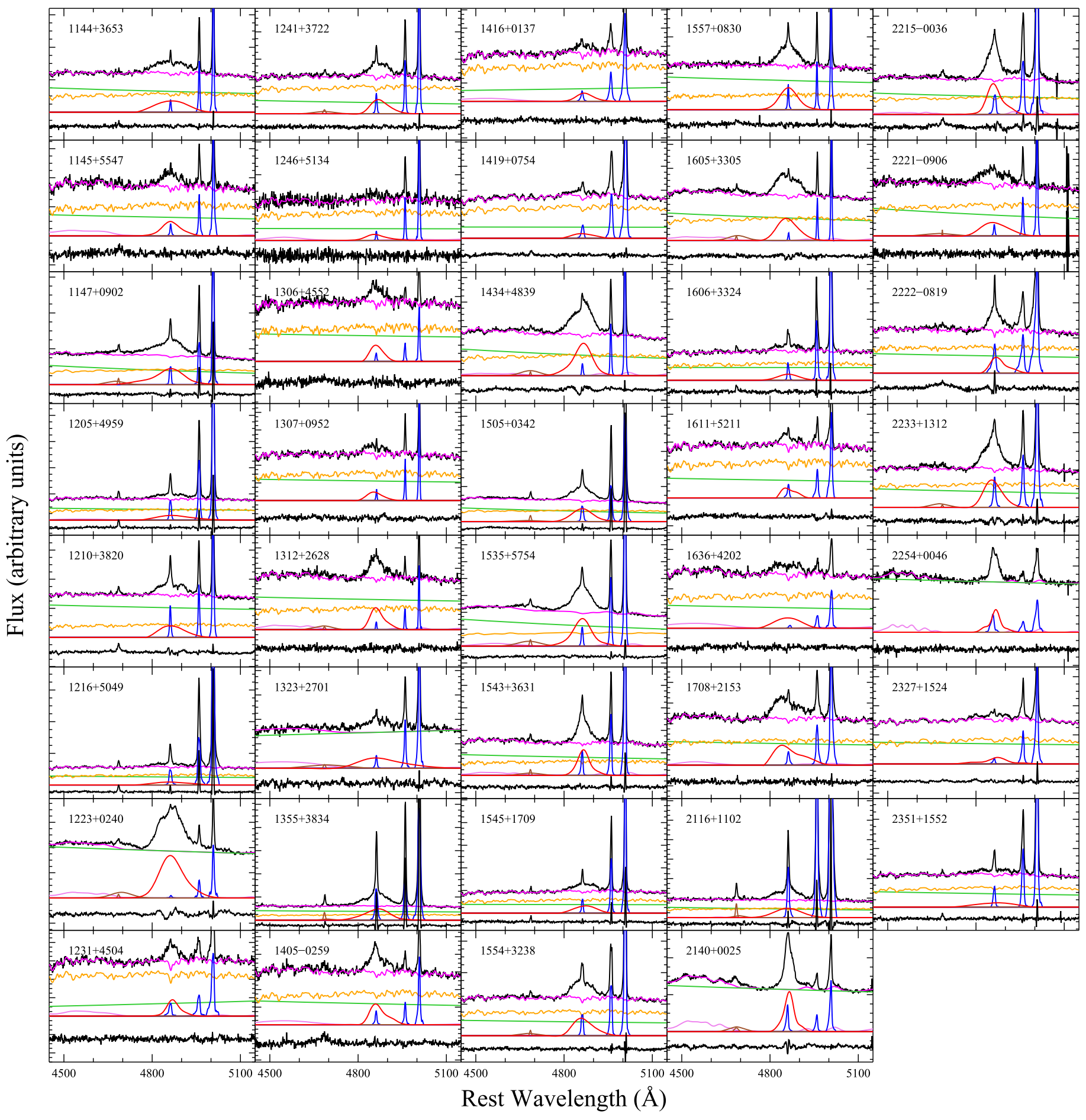

Figure 8. Same as Figure 7.

In the lower right panel of Figure 2, the broad $\mathrm{H} \beta$ to narrow $\mathrm{H} \beta$ peak flux ratio varies quite a bit between SDSS and Keck spectra $(1.26 \pm 0.12)$. This reflects the change in the broad $\mathrm{H} \beta$ emission line, and as a consequence, Seyfert type. On average, the broad $\mathrm{H} \beta$ line is stronger in the SDSS spectra than in the Keck spectra, most likely due the selection bias. The change in peak flux ratio can be used as an independent way to classify Seyfert-type transitions, as discussed in Section 4.1.
Reverberation mapping studies have revealed an anticorrelation between broad $\mathrm{H} \beta$ width and luminosity (Denney et al. 2009; Park et al. 2012; Barth et al. 2015), which is attributed to the relation between ionizing flux and the local reprocessing efficiency of the BLR gas: the $\mathrm{H} \beta$ reprocessing efficiency is greatest in the outer part of the BLR where the flux from the continuum is lower (Korista \& Goad 2004; Goad \& Korista 2014). Therefore, higher levels of continuum luminosity lead to an increase in emissivity-weighted BLR radius. This 


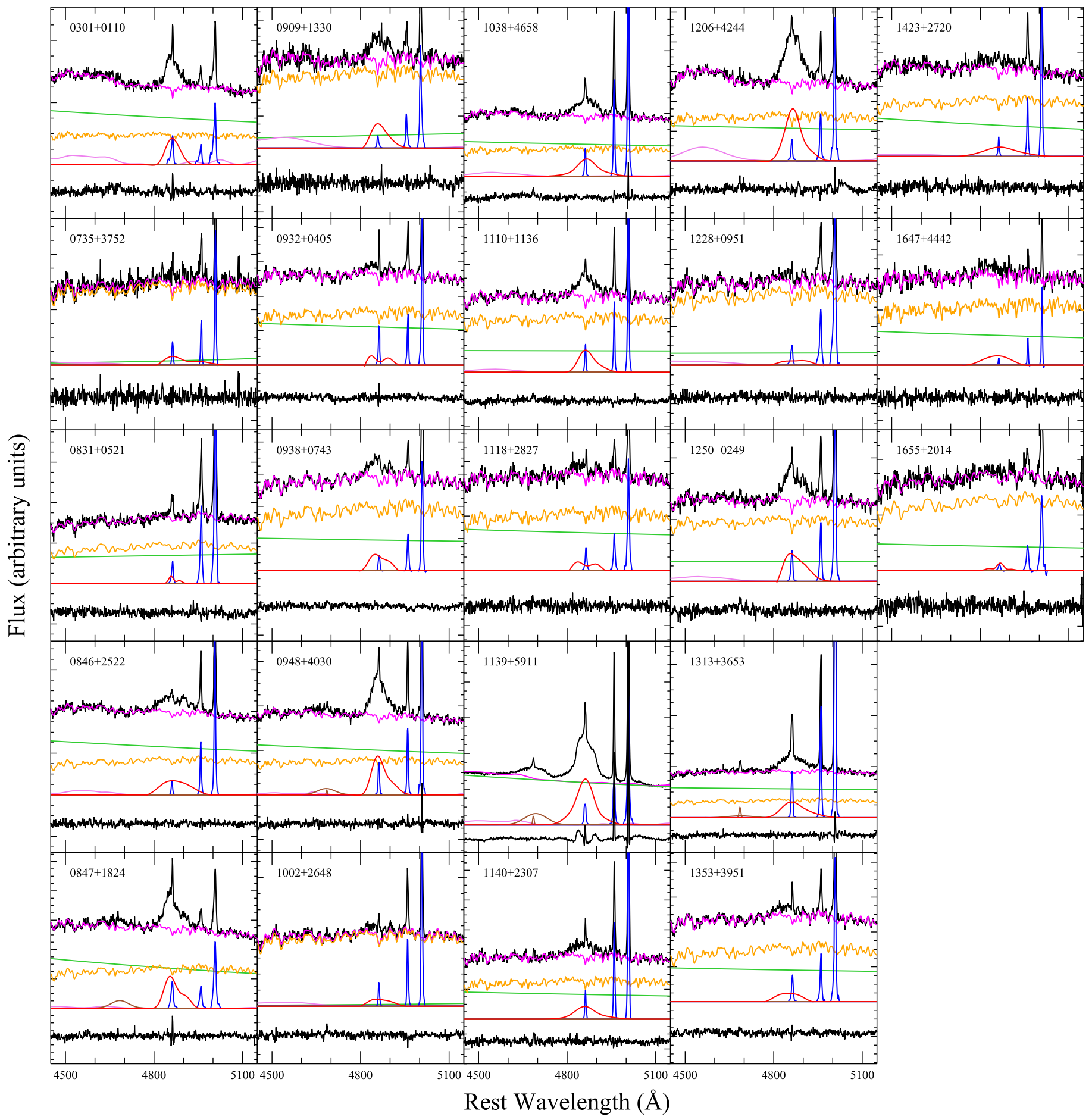

Figure 9. Same as Figure 7, but for 23 objects not included in Bennert et al. (2015).

so-called breathing effect increases flux for low-velocity line core relative to high-velocity wings, which makes the line profile narrower. BLR breathing occurs on short timescales of days to weeks in response to AGN continuum variations. However, our data do not generally support this special kind of line variability.

Variable accretion and/or variable obscuration are considered the two main causes for a type change. As discussed above, to distinguish between them, the variability in X-ray, UV, and optical is typically studied. However, we do not have X-ray data concurring with the SDSS and Keck spectra. Figures 3-6 show a qualitative comparison between the change in $\mathrm{H} \beta$ and powerlaw, overlaying the unsubtracted spectra of SDSS and Keck for each object, scaled to [O III]. There are 41/102 ( 40\%) objects that have a stronger power-law emission in Keck than in SDSS; for $17 / 102(\sim 17 \%)$ it is the other way around, and $44 / 102$ 


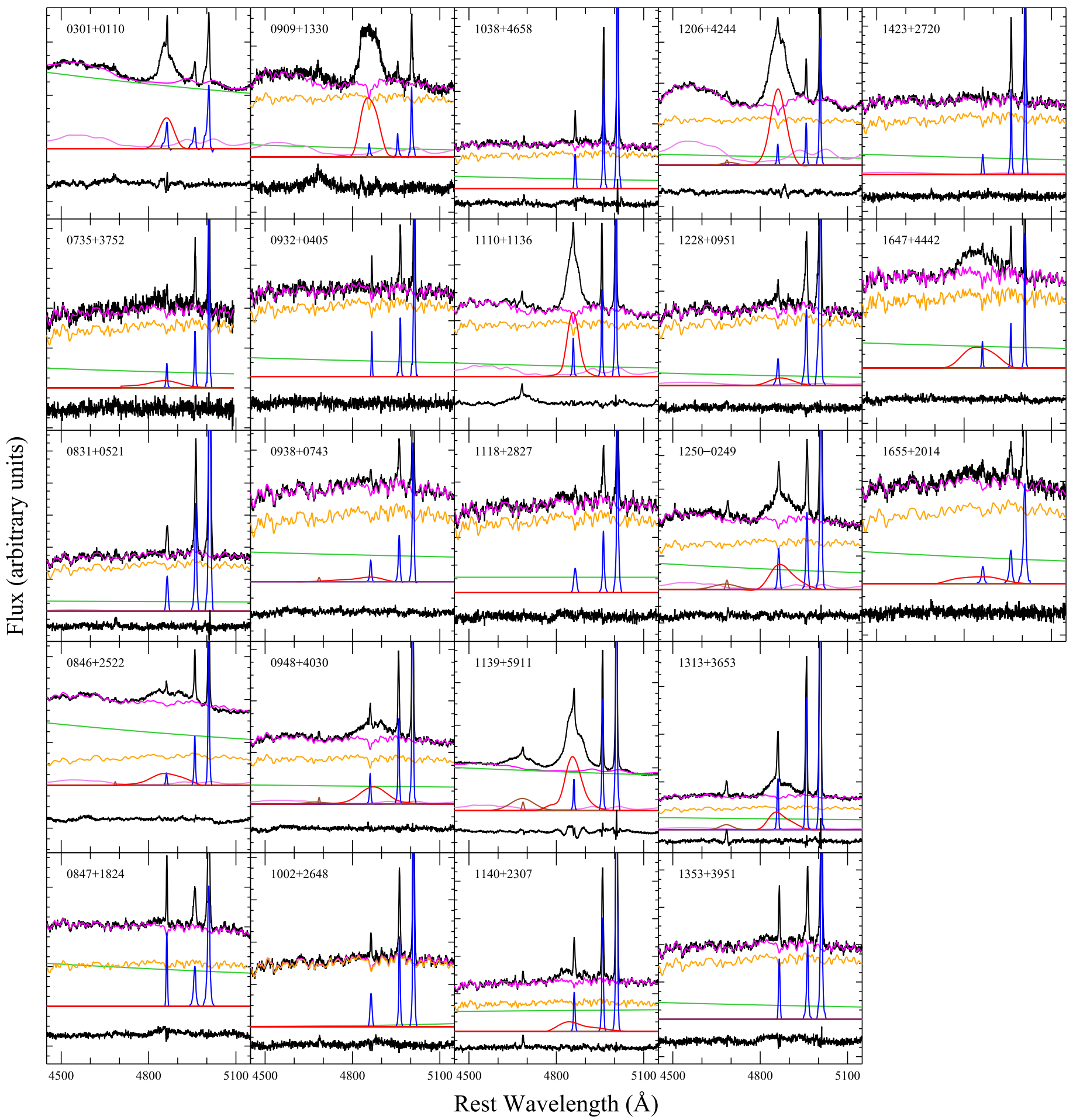

Figure 10. Same as Figure 9 for spectra gathered from Keck and not included in Bennert et al. (2015).

( $\sim 43 \%)$ show no notable change. The higher fraction of nonstellar (power-law) continuum in the Keck spectra is explainable by the smaller slit and sharper seeing.

In other words, both the broad $\mathrm{H} \beta$ emission line as well as the power-law continuum vary between the two set of spectra. However, when attempting to quantify those changes, we do not find them to be directly correlated. This is not too surprising, since any variation between broad $\mathrm{H} \beta$ emission and power-law continuum are offset in time depending on the time- lag of a given object and would not show up at the same time in a single-epoch spectrum (see e.g., Figures 7 and 8 in Park et al. 2012).

Aperture effects may play a role in our results: Keck spectra $\left(1^{\prime \prime} \times 1^{\prime \prime}\right.$ square), given the seeing, only include the unresolved emission from BLR, AGN power-law continuum, and NLR, while the SDSS spectra (1".5 radius circular fiber) may additionally include more extended NLR flux. However, any aperture effect would artificially boost the ratio of broad-to- 

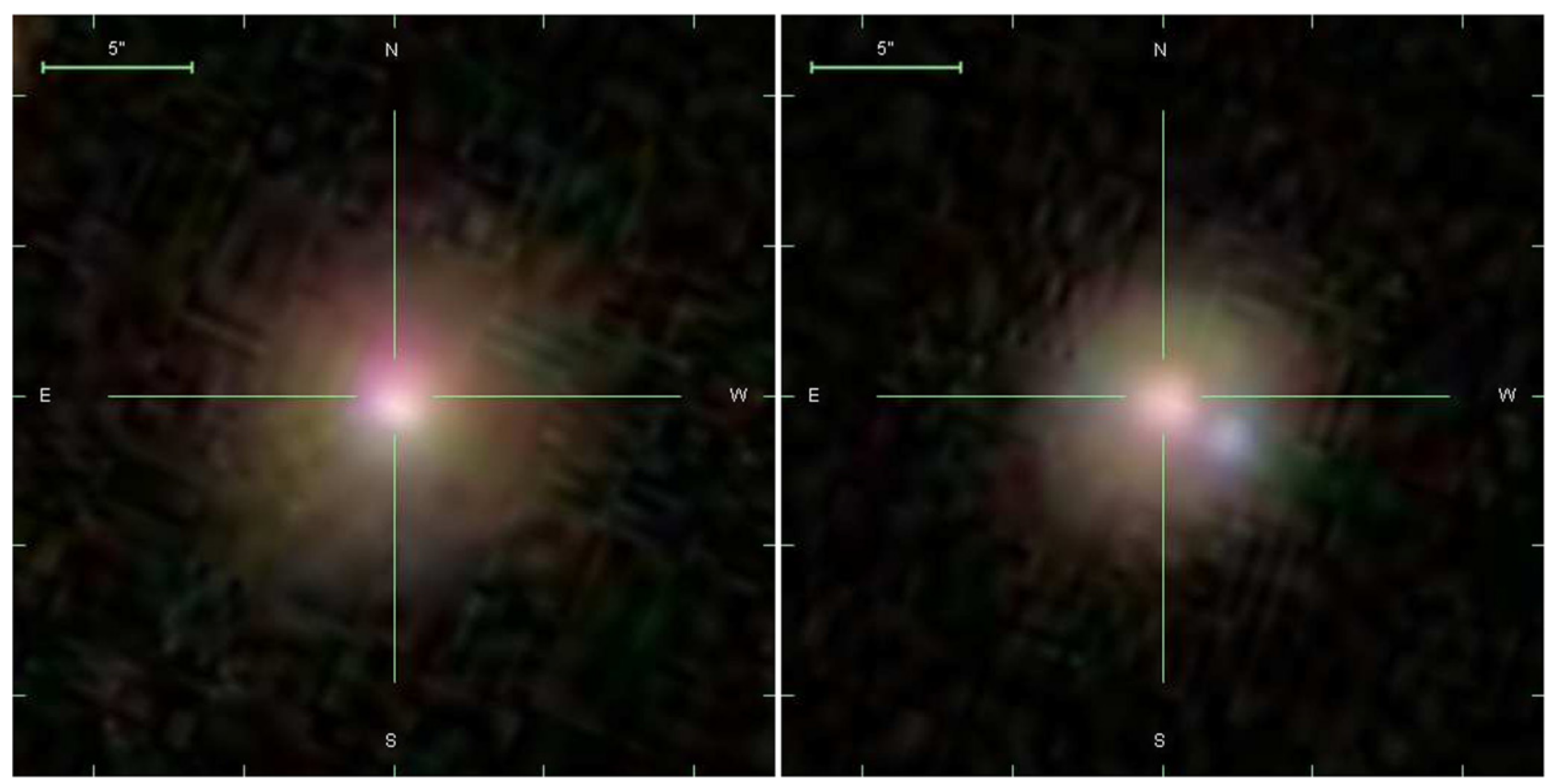

Figure 11. SDSS multi-color image for $0847+1824$ (left) taken on 2004 December 13, roughly a year before the SDSS spectrum, and $1038+4658$ (right) taken on 2002 February 08, roughly 10 months before the SDSS spectrum. For $0847+1824$, there is extended emission offset $\sim 1^{\prime \prime}$ to the northeast of the galaxy center. For $1038+4658$, an emission blob can be seen $\sim 2$ ". 5 to the southwest of the galaxy center. In both cases, the extended emission might have been missed in the Keck spectra due to the smaller width longslit (with a position angle not covering the emission), but included in the $3^{\prime \prime}$ fiber of SDSS.

Table 4

Extreme Seyfert-type Changes

\begin{tabular}{|c|c|c|c|c|}
\hline $\begin{array}{l}\text { Object } \\
\text { (1) }\end{array}$ & $\begin{array}{c}\text { Class. } \\
\text { SDSS } \\
(2)\end{array}$ & $\begin{array}{c}\text { Class. } \\
\text { Keck } \\
(3)\end{array}$ & $\begin{array}{c}\text { Class. } \\
\text { Lick } \\
(4)\end{array}$ & $\begin{array}{c}\text { Notes } \\
(5)\end{array}$ \\
\hline $0847+1824$ & 1 & 1.9 & 2 & $\begin{array}{c}\text { Off-centered emission in the SDSS image } \\
\text { Type- } 1 \text { also in the literature spectrum taken } \sim 1.8 \text { years before SDSS }\end{array}$ \\
\hline $1038+4658$ & 1.5 & 1.9 & 1.9 & Off-centered blob in the SDSS image \\
\hline $1423+2720$ & 1.5 & 1.9 & 1.8 & Low $\mathrm{S} / \mathrm{N}$ in the SDSS spectrum \\
\hline
\end{tabular}

Note. Column (1): Object (for more details see Table 2). Column (2): Seyfert-type classification based on SDSS spectrum. Column (3): Seyferttype classification based on Keck spectrum. Column (4): Seyfert-type classification based on Lick spectrum. Column (5): Notes (see the text for further discussion).

Table 5

Comparison between SDSS and Keck

\begin{tabular}{lc}
\hline \hline $\begin{array}{l}\text { Data } \\
(1)\end{array}$ & $\begin{array}{c}\text { Average } \\
(2)\end{array}$ \\
\hline$\sigma_{\mathrm{H} \beta}$ & $1.07 \pm 0.03$ \\
$\mathrm{FHWM}$ & $1.08 \pm 0.03$ \\
$\mathrm{H} \beta_{\text {narrow }} /[\mathrm{O}$ III] & $1.04 \pm 0.03$ \\
$\mathrm{H} \beta_{\text {broad }} / \mathrm{H} \beta_{\text {narrow }}$ & $1.26 \pm 0.12$ \\
\hline
\end{tabular}

Note. Column (1): comparison between values derived from the SDSS spectra vs. those derived from the Keck spectra for quantities listed in this column. Column (2): average and scatter.

narrow flux in Keck spectra (both peak and integrated) compared with SDSS spectra, since Keck spectra are restricted to a smaller central area and thus focus on the unresolved emission. Thus, aperture effects cannot explain the opposite trend that we are observing in the majority of objects, namely that we observe less broad $\mathrm{H} \beta$ in the Keck spectra.

We note that a few objects (e.g., 0909+1330, 1312+2628, $1708+2153$, and $2140+0025$ ) show significantly stronger blue continuum emission (Figures 3-6). This rise in the blue wavelength range cannot simply be explained by the fact that the Keck spectra were not obtained at parallactic angle since this would have the opposite effect. Similarly, none of the SDSS spectra were taken at large airmasses with the exception of $2140+0025$, observed at an airmass of 1.3 , which could have reduced the blue wavelengths emission artificially for that object in the SDSS spectrum. To further test whether the rise in the blue wavelengths emission in the Keck spectra compared to SDSS is an artifact of Keck flux calibration, we looked at the spatially resolved spectra. The rise in the blue continuum is only present in the central spectra within the seeing limits but not in the outer spectra, suggesting that it is a real trend. A stronger power-law continuum may indeed explain the Seyferttype change observed for $0909+1330$ (from 1.8 in SDSS to 1 in Keck) and $1708+2153$ (from 1.5 in SDSS to 1 in Keck). (Note that the other two objects were classified as Seyfert 1 in 
both spectra). However, as mentioned above, part of this higher fraction of power-law continuum in the Keck spectra can simply be due to the smaller aperture and sharper seeing compared with SDSS.

\subsection{Extreme Seyfert-type Changes}

For three objects in the sample, the broad $\mathrm{H} \beta$ component was very prominent in the SDSS spectra, but decreased significantly and virtually disappeared in the Keck spectra. All three were re-observed with the $3 \mathrm{~m}$ Shane telescope of Lick observatory (Scott 2013). Table 4 summarizes the Seyfert-type changes of these objects. Note that all Keck objects classified conservatively as type- 1.9 could be a type- 2 object; however, the spectra do not extend to $\mathrm{H} \alpha$, so we cannot distinguish between the two. Especially in those cases where the Lick spectrum reveals a type-2 object, it is likely that the object was also a type- 2 in the Keck spectrum. Also note that for 1423 +2720 the SDSS spectrum has a low S/N, making it difficult to model the underlying broad $\mathrm{H} \beta$ line.

The continuum luminosity at $5100 \AA$ was compared for all objects in the sample in order to determine if there was a correlation between AGN luminosity and strongly variable objects, but no correlation was found.

For all objects, we carefully searched the literature for other optical spectra. $0847+1824$ is the only object for which this search was successful: it was previously observed on 02-282004 (MJD 53063) before the SDSS observation, and from that spectrum the AGN was a type-1 Seyfert (Ho \& Kim 2009).

Apart from variable accretion and/or obscuration, we briefly discuss a few other scenarios which could mimic changes in Seyfert type.

(i) Telescope offset: first, we note that apparent Seyfert-type changes could be caused by a slight mispointing of the Keck telescope, missing the AGN core and therefore the (bulk of the) broad emission lines. In all cases, the telescope was pointed at the center of the galaxy (as verified by guide star images), assuming that the AGN resides there. For a couple of extreme objects, the AGN might actually be offset from the center, as evidenced by the SDSS images; we discuss them below. Note that for all objects for which the AGN core and BLR coincide with the center of the galaxy, the possibility of missing the BLR emission due to a telescope offset is negligible, given the seeing $\left(\sim 1^{\prime \prime}\right.$ for the Keck observations) and the slit width used ( $1^{\prime \prime}$, matching the typical seeing), as verified by standard-star observations. Keck telescope guiding is also much more accurate than $1^{\prime \prime}$.

(ii) Galaxy mergers and/or recoiling SMBH: in the course of ongoing galaxy mergers, the AGN can appear offset from the apparent center of the merging system. It is therefore possible that an off-center AGN was captured by the wider, circular SDSS aperture, but missed during subsequent observation by the narrower Keck slit that was aligned along the major axis of the galaxy and centered on the galactic nucleus. Alternatively, a rare gravitational wave recoil following the final coalescence of two SMBHs in a merger can remove the newly formed single SMBH from the center of its host galaxy (e.g., see the review by Sperhake 2015). The accretion disk and BLR would remain bound and a recoiling SMBH would therefore appear as an AGN offset from the core of its host galaxy (Komossa 2012), again leading to the possibility of missing the (bulk of the) BLR in the narrower (and rectangular) Keck slit.
With the exception of a few cases (4/102, see Bennert et al. 2015); there are no signs for merger activity in the sample. However, we caution that merger signatures such as faint tidal tails might easily be missed in the low S/N SDSS images. Objects $0847+1824$ and $1038+4658$ show extended emission in the SDSS multi-color images, offset from the galaxy center (Figure 11), which might indicate the presence of an ongoing merger. While we cannot exclude the possibility that these are off-center AGNs, it is statistically unlikely to have off-centered AGNs in such a small sample, and follow-up spectroscopy would be needed to test such a scenario further. We note in passing that $0847+1824$ seems to show a small kinematic offset between its narrow and broad $\mathrm{H} \beta$ line (with the broad $\mathrm{H} \beta$ line being blueshifted by $\sim 100 \mathrm{~km} \mathrm{~s}^{-1}$ ), which is, however, most likely mimicked by the asymmetric broad-line profile.

(iii) Supernovae: a SN IIn has many of the same spectral features as a Seyfert galaxy (Filippenko 1997). A nuclear supernova could have therefore mimicked the presence of an AGN. However, supernova spectra including the narrow emission lines evolve rapidly, and we do not see any other signs of dramatic changes in the continuum and narrow emission lines.

(iv) Stellar tidal disruption event: stars can be tidally disrupted and accreted by SMBHs, producing a luminous accretion flare (e.g., Rees 1988). If these occur in a gas-rich environment, broad and narrow emission lines can be temporarily excited. While a few candidate events for this process have been identified recently from SDSS (e.g., Komossa et al. 2008), these events are rare and unlikely to occur in our small sample. In particular, we have checked the long-term Catalina lightcurves of all three sources and none show the characteristic lightcurve decline expected for a typical tidal disruption event.

We are left with mild changes in accretion or extinction as the most likely explanation for the three changing look AGN in our sample. Future spectroscopic monitoring of emission line and continuum changes will enable us to distinguish between both possibilities. (Note that a difference in aperture between Keck and SDSS cannot explain the extreme Seyfert-type changes we observe in these three objects since it would have the opposite effect.)

\subsection{Comparison with the Catalina Sky Survey}

To further shed light on the causes for the observed variability, we considered the optical lightcurves in the Catalina Sky Survey (CSS) (Drake et al. 2009). With the exception of $1104+4334$ and $1206+4244$, all objects in our sample are in the CSS archive. (Note that $1605+3305$ is in the archive, but it does not have a lightcurve available.) For most objects, CSS lightcurves begin after the SDSS observations, but extend past the time of the Keck and Lick observations; the lightcurves start and end at approximately 53500-56500 MJD ( $\sim 3000$ days). The lightcurves of seven objects (0310-0049, $0904+5536, \quad 1147+0902,1355+3834,1434+4839,1535$ +5754 , and $1557+0830)^{9}$ reveal large variability $(\sim 0.5-1 \mathrm{mag}$ in one object) over timescales from days to months to years. None of these objects are among our extreme subset of objects (see Table 2 for Seyfert types).

\footnotetext{
9 Note that $0301+0110$ shows a highly variable lightcurve, but upon further inspection it becomes clear that that is an artifact of a nearby bright star.
} 
Since CSS photometry is aperture-based (Drake et al. 2009), we cannot exclude that a variable seeing can mimic variability since more or less of the host galaxy would be included in an aperture centered on the AGN. However, especially the extreme variability in these seven objects is unlikely to be purely a seeing effect. Independent analysis, which is beyond the scope of this paper, is needed to further confirm the observed variability.

\section{SUMMARY}

In this paper, we study the broad $\mathrm{H} \beta$ emission line variability in a sample of 102 local Seyfert 1 galaxies, selected from SDSS and re-observed three to nine (on average $6.4 \pm 1.8$ ) years later with LRIS on the $10 \mathrm{~m}$ Keck-I telescope.

In the three to nine year time frame between observations, $67 / 102(\sim 66 \%)$ objects show at least some form of variability of either width and/or strength of the broad $\mathrm{H} \beta$ line. For 39/ $102(\sim 38 \%)$ objects, this variability is significant enough to result in a change in Seyfert type, following the standard Seyfert classification scheme. There is no correlation between the time between observations and the degree of the observed Seyfert-type transition, implying that the transitions happen on shorter timescales. Short-time variability on the scale of days and weeks is known for low-mass AGNs from reverberation mapping. Almost all objects (99/102) were observed as part of the CSS with 7/102 ( 7\%) displaying significant variability on timescales of days to weeks.

Three ( $3 \%)$ objects are extreme cases for which the broad $\mathrm{H} \beta$ component almost completely disappears. We discuss possible origins for these transitions. For two of these objects $(0847+1824$ and $1038+4658)$, SDSS images reveal extended emission off-centered from the galaxy center which could have been included in the SDSS spectra, but missed by the smallerarea Keck slit centered on the galaxy.

The study presented here is the first to provide statistical information on the frequency and strength of $\mathrm{H} \beta$ line variability in a sample of low-redshift Seyfert galaxies.

We thank the anonymous referee for valuable comments that helped to improve our paper. We thank Aaron Barth and Bernd Husemann for helpful discussions, Luis Ho for providing additional data, and William C. Keel for data reduction of the Lick spectra. J.N.R., M.C., and V.N.B. gratefully acknowledge the assistance from a National Science Foundation (NSF) Research at Undergraduate Institutions (RUI) grant AST1312296. Note that the findings and conclusions presented in this paper do not necessarily represent the views of the NSF. V. N.B., B.S., and S.K. thank the Kavli Institute for Theoretical Physics (Santa Barbara) for their hospitality and support; the KITP is supported by NSF grant no. NSF PHY11-25915. D.P. acknowledges support through the EACOA Fellowship from The East Asian Core Observatories Association, which consists of the National Astronomical Observatories, the Chinese Academy of Science (NAOC), the National Astronomical Observatory of Japan (NAOJ), the Korean Astronomy and Space Science Institute (KASI), and the Academia Sinica Institute of Astronomy and Astrophysics (ASIAA). This research has made use of the Dirac computer cluster at the California Polytechnic State University in San Luis Obispo, maintained by Dr. Brian Granger and Dr. Ashley Ringer McDonald. Data presented in this thesis were obtained at the
W. M. Keck Observatory, which is operated as a scientific partnership between Caltech, the University of California, and NASA. The W. M. Keck Observatory was made possible by the generous financial support of the W. M. Keck Foundation. The authors recognize and acknowledge the very significant cultural role and reverence that the summit of Mauna Kea has always had within the indigenous Hawaiian community. We are most fortunate to have the opportunity to conduct observations from this mountain. This research has made use of the public archive of the SDSS and the NASA/IPAC Extragalactic Database (NED), which is operated by the Jet Propulsion Laboratory, California Institute of Technology, under contract with the National Aeronautics and Space Administration. The CSS survey is funded by the National Aeronautics and Space Administration under grant no. NNG05GF22G issued through the Science Mission Directorate Near-Earth Objects Observations Program. The CRTS survey is supported by the U.S. National Science Foundation under grants AST-0909182 and AST-1313422.

Facilities: Keck: I (LRIS), Lick: Shane $3 \mathrm{~m}$ Kast spectrograph.

\section{REFERENCES}

Adelman-McCarthy, J. K., Agueros, M. A., Allam, S. S., et al. 2008, ApJ, 175,297

Antonucci, R. R. J., \& Cohen, R. D. 1983, ApJ, 271, 564

Arcavi, I., Gal-Yam, A., Sullivan, M., et al. 2014, ApJ, 793, 38

Aretxaga, I., Joguet, B., Kunth, D., Melnick, J, \& Terlevich, R. J. 1999, ApJL, 519, L123

Barth, A. J., Bennert, V. N., Canalizo, G., et al. 2015, ApJS, 217, 26

Bennert, V. N., Auger, M. W., Treu, T., Woo, J. H., \& Malkan, M. A. 2011, ApJ, 726, 59

Bennert, V. N., Treu, T., Auger, M. W., et al. 2015, ApJ, 809, 20

Bentz, M. C., Denney, K. D., Cackett, E. M., et al. 2007, ApJ, 662, 205

Bentz, M. C., Denney, K. D., Grier, C. J., et al. 2013, ApJ, 767, 149

Bochkarev, N. G. 2006, ASPC, 360, 285

Boroson, T. A., \& Green, R. F. 1992, ApJS, 80, 109

Denney, K. D., Peterson, B. M., Dietrich, M., Vestergaard, M., \& Bentz, M. C. 2009, ApJ, 692, 246

Denney, K. D., Rosa, G. D., Croxall, K., et al. 2014, ApJ, 796, 134

Dimitrijević, M. S., Popović, L. Č., Kovačević, J., Dačić, M., \& Ilić, D. 2007 MNRAS, 374, 1181

Drake, A. J., Djorgovski, S. G., Mahabal, A., et al. 2009, ApJ, 696, 870

Elitzur, M., Ho, L. C., \& Trump, J. R. 2014, MNRAS, 438, 3340

Eracleous, M., \& Halpern, J. P. 2001, ApJ, 554, 240

Ferrarese, L., \& Merrit, D. 2000, ApJL, 539, L9

Filippenko, A. V. 1997, ARA\&A, 35, 309

Gebhardt, K., Bender, R., Bower, G., et al. 2000, ApJL, 539, L13

Goad, M. R., \& Korista, K. T. 2014, MNRAS, 444, 43

Goodrich, R. W. 1989, ApJ, 340, 190

Graham, A. 2016, in Galactic Bulges, ed. E. Laurikainen, R. F. Peletier, \& D. A. Gadotti, Vol. 418 (Berlin: Springer), 263

Harris, C. E., Bennert, V. N., Auger, M. W., et al. 2012, ApJS, 201, 29

Hicks, E. K. S., \& Malkan, M. A. 2008, ApJS, 174, 31

Ho, L. C., \& Kim, M. 2009, ApJS, 184, 398

Kaspi, S., Maoz, D., Netzer, H., et al. 2005, ApJ, 629, 61

Kollatschny, W., \& Fricke, K. J. 1985, A\&A, 146, L11

Komossa, S. 2012, AdAst, 2012, 364973

Komossa, S., Zhou, H., Wang, T., et al. 2008, ApJL, 678, L13

Korista, K. T., \& Goad, M. R. 2004, ApJ, 606, 749

Kormendy, J., \& Ho, L. C. 2013, ARAA, 51, 511

Kormendy, J., \& Richstone, D. 1995, ARA\&A, 33, 581

Kovacevic, J., Popovic, L. C., \& Dimitrijevic, M. S. 2010, ApJS, 189, 15

LaMassa, S. M., Cales, S., Moran, E. C., et al. 2015, ApJ, 800, 144

Leighly, K. M., Cooper, E., Grupe, D., et al. 2015, ApJL, 809, L13

Lyutyi, V. M., Oknyanskii, V. L., \& Chuvaev, K. K. 1984, SvAL, 10, 335

MacLeod, C. L., Ross, N. P., Lawrence, A., et al. 2016, MNRAS, 457, 389

Magorrian, J., Tremaine, S., Richstone, D., et al. 1998, AJ, 115, 2285

McGill, K. L., Woo, J. H., Treu, T., \& Malkan, M. A. 2008, ApJ, 673, 703

Merloni, A., Dwelly, T., Salvato, M., et al. 2015, MNRAS, 452, 69 
Nicastro, F. 2000, ApJL, 530, L65

Osterbrock, D. E. 1977, ApJ, 215, 733

Osterbrock, D. E. 1981, ApJ, 249, 462

Osterbrock, D. E. 1989, Astrophysics of Gaseous Nebulae and Active Galactic Nuclei (Mill Valley, CA: Univ. Science Books)

Osterbrock, D. E., \& Koski, A. T. 1976, MNRAS, 176, 61

Pancoast, A., Brewer, B. J., Treu, T., et al. 2014, MNRAS, 445, 3073

Park, D., Woo, J. H., Bennert, V. N., et al. 2015, ApJ, 799, 164

Park, D., Woo, J. H., Treu, T., et al. 2012, ApJ, 747, 30

Parker, M. L., Fabian, A. C., Matt, G., et al. 2015, MNRAS, 447, 72

Penston, M. V., \& Perez, E. 1984, MNRAS, 211, 33

Peterson, B., Denney, K. D., De Rosa, G., et al. 2013, ApJ, 779, 109

Rees, M. J. 1988, Natur, 333, 523

Rosenblatt, E. I., Malkan, M. A., Sargent, W. L. W., \& Readhead, A. C. S. 1994, ApJS, 93, 73

Ruan, J. J., Anderson, S. F., Cales, S. L., et al. 2015, ApJ, submitted (arXiv:1509.03634)

Runnoe, J. C., Cales, S., Ruan, J. F., et al. 2016, MNRAS, 455, 1691
Scott, B. 2013, Time Variation of the Broad $\mathrm{H} \beta$ and $\mathrm{H} \alpha$ Emission Lines in Active Galactic Nuclei, Cal Poly Digital Commons

Shapovalova, A. I., Popovic, L. C., Burenkov, A. N., et al. 2010, A\&A, 509, A106

Shappee, B. J., Prieto, J. L., Grupe, D., et al. 2014, ApJ, 788, 48

Shen, J., Vanden Berk, D. E., Schneider, D. P., \& Hall, P. B. 2008, ApJ, 135,928

Shen, Y., Richards, G. T., Strauss, M. A., et al. 2011, ApJS, 194, 45

Sperhake, U. 2015, ASSP, 40, 185

Storchi-Bergmann, T., Baldwin, J. A., \& Wilson, A. S. 1993, ApJL, 410, L11

Tohline, J. E., \& Osterbrock, D. E. 1976, ApJL, 210, L117

Trippe, M. L., Crenshaw, D. M., Deo, R., \& Dietrich, M. 2008, AJ, 135, 2048

Valdez, F., Gupta, R., Rose, J. A., Singh, H. P., \& Bell, D. J. 2004, ApJS, 152,251

van der Marel, R. P., Cretton, N., de Zeeuw, P. T., \& Rix, H. 1998, ApJ, 493, 613 van der Marel, R. P., \& Franx, M. 1993, ApJ, 407, 525

Wandel, A., Peterson, B. M., \& Malkan, M. A. 1999, ApJ, 526, 579

Woo, J. H., Treu, T., Malkan, M. A., \& Blanford, R. D. 2006, ApJ, 645, 900 